\title{
FROBENIUS RECIPROCITY AND EXTENSIONS OF NILPOTENT LIE GROUPS
}

\author{
JEFFREY FOX
}

\begin{abstract}
In $\S 1$ we use $C^{\infty}$-vector methods, essentially Frobenius reciprocity, to derive the Howe-Richardson multiplicity formula for compact nilmanifolds. In $\$ 2$ we use Frobenius reciprocity to generalize and considerably simplify a reduction procedure developed by Howe for solvable groups to general extensions of nilpotent Lie groups. In $\$ 3$ we give an application of the previous results to obtain a reduction formula for solvable Lie groups.
\end{abstract}

Introduction. In the representation theory of rings the fact that the tensor product functor and Hom functor are adjoints is of fundamental importance and at the time very simple and elegant. When this adjointness property is translated into a statement about representations of finite groups via the group ring, the classical Frobenius reciprocity theorem emerges. From this point of view, Frobenius reciprocity, aside from being a useful tool, provides a very natural explanation for many phenomena in representation theory.

When $G$ is a Lie group and $\Gamma$ is a discrete cocompact subgroup, a main problem in harmonic analysis is to describe the decomposition of $\operatorname{ind}_{\Gamma}^{G}(1)$ - the quasiregular representation. When $G$ is semisimple the problem is enormously difficult, and little is known in general. However, if $G$ is nilpotent, a rather detailed description of $\operatorname{ind}_{\Gamma}^{G}(1)$ is available, thanks to the work of Moore, Howe, Richardson, and Corwin and Greenleaf [M, H-1, R, C-G]. If $G$ is solvable Howe has developed an inductive method of describing ind $\Gamma_{\Gamma}^{G}(1)[\mathbf{H}-2]$. In all of the above cases, the basic tools used are the ingredients of the now standard Mackey normal subgroup analysis, and the result obtained might be viewed as saying that a version of the classical Frobenius reciprocity theorem holds in each instance. Of course, it would be desirable to obtain these results as a consequence of a Frobenius reciprocity theorem.

When studying semisimple Lie groups, the authors of $[\mathbf{G}]$ recognize the correct formulation of Frobenius reciprocity for Lie groups and provide a proof that depends on the rigid structural aspects of semisimple Lie groups and their representations. This structural property lets one replace an analytic problem with an algebraic one that can be dealt with. In [Po], Poulsen saw what the core of the difficulty was and using elliptic regularity arguments solved the technical analytic problem in general. From here it was a quick step in proving very general Frobenius reciprocity theorems for Lie groups, as Penney did in [P-1] and [P-2].

Received by the editors May 4, 1984 and, in revised form, February 20, 1986.

1980 Mathematics Subject Classification. Primary 22E25; Secondary 22E40. 
In $\$ 1$ we use Penney's Frobenius reciprocity theorem to give a new proof of the Howe-Richardson multiplicity formula which is based entirely on $C^{\infty}$-vector methods. Richardson noticed that his lift maps constructed in [R] already solved the multiplicity problem for the three-dimensional Heisenberg group, and we essentially give an extension of that observation to all connected simply connected nilpotent Lie groups. In $\$ 2$ we develop an inductive procedure for determining the structure of $\operatorname{ind}_{\Gamma}^{G}(1)$ that follows closely the Mackey normal subgroup analysis and Howe's reduction procedure in [H-2]. By exploiting Penney's Frobenius reciprocity theorem, our results provide a great simplification and a generalization of Howe's Theorem 2.1 of [H-2].

In $\$ 3$ we give an application of the previous results to solvable Lie groups. By using Penney's canonical objects we reduce the multiplicity problem to the following special case. Let $N \subseteq G$ be the nilradical of $G$. Let $\rho$ be an irreducible representation of $G$ occurring in $L^{2}(G / \Gamma)$. Then we can assume

(a) $\left.\rho\right|_{N}$ is a multiple of an irreducible representation $\pi$ of $N$ (so $G$ stabilizes $\pi$ ).

(b) $\pi$ is square integrable modulo its kernel.

Though this problem is much more difficult to solve than when $G$ is an abelian extension of a Heisenberg group (see [H-2]) it has the advantage that the connection with the orbit parameterization of Auslander and Kostant is left intact. Also, we have stated our result in terms of multiplicities, but if we had used the results of Corwin and Greenleaf on intertwining operators instead of Howe's multiplicity formula (cf. Theorem 3.2), it is clear that Theorem 3.2 would give a (manageable) way of computing constructible subspaces, primary projection, etc. for solvmanifolds once the corresponding problem has been settled for the special case.

Parts of this paper appeared in the author's dissertation, and it is a pleasure to thank my advisor, Calvin Moore, for his support and guidance.

0. Let $G$ be a Lie group with Lie algebra $\mathbf{G}$. We let $\underline{\mathbf{U}}(\mathbf{G})$ be the universal enveloping algebras of $\mathbf{G}$. By a representation of $G$ we will mean, unless otherwise noted, a continuous unitary representation $\pi$ acting on a Hilbert space $H(\pi)$. A vector $v \in H(\pi)$ is called a $C^{\infty}$-vector if the mapping $g \rightarrow \pi(g) v$ is a $C^{\infty}$-mapping. We denote by $H^{\infty}(\pi)$ the subspace of $C^{\infty}$-vectors. For $X \in \mathbf{G}$ and $u \in H^{\infty}(\pi)$ define $\pi(X) u$ by

$$
\pi(X) u=\left.\frac{d}{d t} \pi(\exp (t X)) \cdot u\right|_{t=0} .
$$

The mapping $X \rightarrow \pi(X)$ gives rise to a Lie algebra representation of $G$ on $H^{\infty}(\pi)$, so it extends to a representation of $\underline{\mathbf{U}}(\mathbf{G})$ on $H^{\infty}(\pi)$. If $a \in \underline{\mathbf{U}}(\mathbf{G})$ and $v \in H^{\infty}(\pi)$ define $\rho_{a}(x)=\|\pi(a) \cdot v\|$. Then $\left\{\rho_{a} \mid a \in \underline{\mathbf{U}}(\mathbf{G})\right\}$ defines a family of seminorms on $H^{\infty}(\pi)$ which gives $H^{\infty}(\pi)$ the structure of a Fréchet space. If we restrict the action of $\pi$ to $H^{\infty}(\pi)$ then we obtain a $C^{\infty}$-representation of $G$ on $H^{\infty}(\pi)$. We will also denote this representation by $\pi$. Let $H^{-\infty}(\pi)$ be the topological dual space of $H^{\infty}(\pi)$. Then there is a natural action of $G$ on $H^{-\infty}(\pi)$ given by duality. If we also denote this action by $\pi$ we have for $D \in H^{\infty}(\pi), v \in H^{\infty}(\pi)$

$$
(\pi(g) D)(v)=D\left(\pi\left(g^{-1}\right) v\right) .
$$


Let $\Gamma \subseteq G$ be a cocompact subgroup of $G$ and $\alpha$ a finite dimensional representation of $\Gamma$. If $\rho=\operatorname{ind}_{\Gamma}^{G}(\alpha)$ then it is well known that $\rho$ decomposes into a direct sum of irreducible representations of $G$, each occurring with finite multiplicity $[G]$. Theorem 5.1 of [Po] characterizes the $C^{\infty}$-vectors for an induced representation. If we apply it to $\rho$ we have the following lemma.

LEMMA 0.1. Let $\Gamma \subseteq G$ be a cocompact subgroup of $G$ and $\alpha$ a finite dimensional representation of $\Gamma$ on $H(\alpha)$. If $\rho=\operatorname{ind}_{\Gamma}^{G}(\alpha)$ then

$$
H^{\infty}(\rho)=\left\{f: G \rightarrow H(\alpha) \mid f \text { is a } C^{\infty} \text {-function and } f(g \gamma)=\alpha\left(\gamma^{-1}\right) \cdot f(g)\right\}
$$

with the topology being uniform convergence of all derivatives on compact subsets of $G$.

Using this we can write out a version of Frobenius reciprocity that is formally the same as for finite groups. Thus, if $\pi$ is an irreducible representation of $G$ which occurs in $\rho$ there exists a nonzero bounded intertwining map $T: H(\pi) \rightarrow H(\rho)$. We will also denote by $T$ the restriction of $T$ to $H^{\infty}(\pi)$. Thus we have a continuous map T: $H^{\infty}(\pi) \rightarrow H^{\infty}(\rho)$. Now construct a map $D_{T}: H^{\infty}(\pi) \rightarrow H(\alpha)$ by $D_{T}(v)=$ $T(v)(e)$. Now

$$
T(\pi(\gamma) v)(e)=\rho(\gamma) T(v)(e)=T(v)\left(\gamma^{-1}\right)=\alpha(\gamma) T(v)(e),
$$

so we have $D_{T} \in \operatorname{Hom}_{\Gamma}\left(H^{\infty}(\pi), H(\alpha)\right.$ ) (where by $\operatorname{Hom}_{\Gamma}\left(H^{\infty}(\pi), H(\alpha)\right.$ ) we mean continuous maps of $H^{\infty}(\pi)$ into $H(\alpha)$ ). Consequently, if we could show $T \rightarrow D_{T}$ is onto, there would be the canonical isomorphism of Frobenius reciprocity. To this end we have the following theorem of Penney [P-1].

THEOREM 0.1. Let $\Gamma$ be a cocompact subgroup of $G, \alpha$ a finite dimensional representation of $\Gamma$, and $\rho=\operatorname{ind}_{\Gamma}^{G}(\alpha)$. Let $\pi$ be an irreducible representation of $G$ and define a map

$$
D: \operatorname{Hom}_{G}(H(\pi), H(\rho)) \rightarrow \operatorname{Hom}_{\Gamma}\left(H^{\infty}(\pi), H(\alpha)\right)
$$

as follows. Given $T \in \operatorname{Hom}_{G}(H(\pi), H(\rho)), v \in H^{\infty}(\pi)$ then $D(T)(v)=T(v)(e)$. The map $D$ is an isomorphism. Furthermore, there is a natural inner product on the space $\operatorname{Hom}_{\Gamma}\left(H^{\infty}(\pi), H(\alpha)\right)$ which we denote by $($,$) . If \langle$,$\rangle is the inner product on$ $H(\pi),\langle,\rangle_{\alpha}$ the inner product on $H(\alpha)$, and $u, v \in H^{\infty}(\pi)$ we have

$$
(D, E) \cdot\langle u, v\rangle=\int_{G / \Gamma}\langle(\pi(g) D)(u),(\pi(g) E)(u)\rangle_{\alpha} d g
$$

REMARK. If we dualize this theorem we get the classical version of Frobenius reciprocity,

$$
\operatorname{Hom}_{G}(H(\pi), H(\rho)) \simeq \operatorname{Hom}_{\Gamma}\left(H(\alpha)^{*}, H^{-\infty}(\pi)\right) .
$$

Suppose $N$ is a connected simply connected nilpotent Lie group with Lie algebra $\underline{\mathbf{N}}$. If $\Gamma \subseteq N$ is a discrete cocompact subgroup of $N$, then $\log (\Gamma)$ contains a basis of $\underline{\mathbf{N}}$, so if we let $\underline{\mathbf{N}}_{Q}=\operatorname{span}_{\mathbf{Q}}(\log (\Gamma)), \underline{\mathbf{N}}_{Q}$ is a rational subspace of $\underline{\mathbf{N}}$ such that $\underline{\mathbf{N}}_{Q} \otimes_{\mathbf{Q}} \mathbb{R}=\underline{\mathbf{N}}$. One says that a group element $g=\exp (X)$ is rational if $X \in \underline{\mathbf{N}}_{Q}$. If $H \subseteq N$ is a connected subgroup with Lie algebra $\underline{\mathbf{H}}$, then $H$ is called rational if $\underline{\mathbf{H}} \cap \underline{\mathbf{N}}_{Q}$ contains a basis of $\underline{\mathbf{H}}$ over $\mathbb{R}$. A very nice discussion of rationality 
properties for nilpotent Lie groups can be found in the appendix of [C-G-1]. We will content ourselves to quoting the facts we will need.

LEMMA 0.2. Let $N$ be a connected simply connected nilpotent Lie group with Lie algebra $\underline{\mathbf{N}}$. Suppose $\Gamma \subseteq N$ is a discrete cocompact subgroup. Then

(a) there is a basis of $\underline{\mathbf{N}}, X_{1}, \ldots, X_{n} \in \underline{\mathbf{N}}_{Q}$ such that the structure constants $C_{i j}^{k}$ of the Lie algebra with respect to this basis are in $\mathbb{Q}$;

(b) let $H$ be a connected subgroup of $N$ with Lie algebra $\underline{\mathbf{H}}$. Then $H \cap \Gamma$ is cocompact in $H$ if and only if $\underline{\mathbf{H}} \cap \underline{\mathbf{N}}_{Q}$ contains a basis for $\underline{\mathbf{H}}$ over $\mathbb{R}$ (i.e. $\underline{\mathbf{H}}$ is a rational subspace of $\underline{\mathbf{N}}_{Q}$ ).

REMARK. Lemma 0.2 (a) implies that $N$ has the structure of an affine algebraic group defined over $\mathbb{Q}$. It should be noted that the notion of a rational element and a rational subgroup in the sense of algebraic groups coincide with our definition here.

1. In this section we want to extend Richardson's observation to all connected simply connected nilpotent Lie groups that the lift maps he constructed in [R] could be used to solve the multiplicity problem for $L^{2}(G / \Gamma)$ when $G$ is a Heisenberg group. To do this we will view his lift maps as elements of $H^{-\infty}(\pi)$ and apply Theorem 0.1 .

Let $G$ be a connected simply connected nilpotent Lie group with Lie algebra $\underline{\mathbf{G}}$. If $H$ is a closed connected subgroup of $G$ then one can find element $X_{1}, \ldots, X_{l} \in \underline{\mathbf{G}}$ such that the map $F: \mathbb{R}^{l} \times H \rightarrow G$ given by $F\left(t_{1}, \ldots, t_{l}, h\right)=$ $\exp \left(t_{1} X_{1}\right) \cdots \exp \left(t_{l} X_{l}\right) \cdot h$ is a diffeomorphism onto $G$. If $\underline{\mathbf{H}}$ is the Lie algebra of $H$, then the set $X_{1}, \ldots, X_{l}$ is called a coexponential basis for $\underline{\mathbf{H}}$ in $\underline{\mathbf{G}}$. Given a coexponential basis of $\underline{\mathbf{H}}$ in $\underline{\mathbf{G}}$ we have a global cross section for $G / H$ that identifies $G / H$ with $\mathbb{R}^{n}$.

Let $\underline{\mathbf{G}}^{*}$ be the dual of $\underline{\mathbf{G}}$ and $f \in \underline{\mathbf{G}}^{*}$. Recall that a subalgebra $\underline{\mathbf{H}} \subseteq \underline{\mathbf{G}}$ is subordinate to $f$ if $\underline{\mathbf{H}}$ is an isotropic subspace for the bilinear form $B_{f}(X, Y)=$ $f([X, Y])$. The subalgebra $\underline{\mathbf{H}}$ is called a polarization for $f$ if $\underline{\mathbf{H}}$ is a maximal totally isotropic subspace for $B_{f}$. If $\underline{\mathbf{H}}$ is a polarization for $f$ let $H=\exp (\underline{\mathbf{H}})$ be the connected subgroup of $G$ with Lie algebra $\underline{\mathbf{H}}$ and define a character of $H$ by the formula $\chi_{f}(\exp (X))=\exp (2 \pi i f(X))$ for all $X \in \underline{\mathbf{H}}$. If $\pi_{f}=\operatorname{ind}_{H}^{G}\left(\chi_{f}\right)$ then $\pi_{f}$ is an irreducible representation of $G$ whose equivalence class depends only on the coadjoint orbit of $f$. Now a simple argument shows that if we pick any coexponential basis for $\underline{\mathbf{H}}$ and use this to identify $G / H$ with $\mathbb{R}^{n}$ then the $C^{\infty}$-vectors for $\pi_{f}$ are exactly the Schwartz functions on $\mathbb{R}^{n}[\mathbf{H}-3$, Proposition 3.3].

Suppose $\Gamma \subseteq G$ is a discrete cocompact subgroup of $G$. Then the following definitions and lemmas can be found in [C-G-1].

DEFinition 1.1. Let $\Gamma$ be a discrete torsion-free nilpotent group. A weak Malcev basis for $\Gamma$ is a set $\left\{d_{1}, \ldots, d_{p}\right\} \subseteq \Gamma$ such that

(i) for any $d \in \Gamma$ there is a decomposition $d=d_{1}^{n_{1}} \cdots d_{p}^{n_{p}}$, where $n_{i} \in \mathbb{Z}$;

(ii) the set $\Gamma_{i}=\left\{d_{1}^{n_{1}} \cdots d_{i}^{n_{i}} \mid n_{j} \in \mathbb{Z}, 1 \leqslant i \leqslant j\right\}$ is a subgroup with $\Gamma_{i-1}$ normal in $\Gamma_{i}$ for $i=1,2, \ldots, p$;

(iii) $\Gamma_{i-1} \backslash \Gamma_{i} \simeq \mathbb{Z}, i=1,2, \ldots, p$. 
DEFINITION 1.2. Let $G$ be a connected simply connected nilpotent Lie group. A weak Malcev basis for $G$ is a set $\left\{X_{1}, \ldots, X_{p}\right\} \subseteq \underline{\mathbf{G}}$ such that

(i) for any $x \in G$, there exists $t_{i} \in \mathbb{R}$ and $1 \leqslant i \leqslant p$, such that $x=$ $\exp \left(t_{1} X_{1}\right) \cdots \exp \left(t_{p} X_{p}\right)$

(ii) the set $G_{i}=\left\{\exp \left(t_{1} X_{1}\right) \cdots \exp \left(t_{i} X_{i}\right) \mid t_{1}, \ldots, t_{i} \in \mathbb{R}\right\}$ is a closed subgroup of $G$ with $G_{i-1}$ normal in $G_{i}$ for $i=1,2, \ldots, p$;

(iii) $G_{i-1} / G_{i} \simeq \mathbb{R}$ for $i=1,2, \ldots, p$.

If $\Gamma \subseteq G$ is a discrete cocompact subgroup of $G$ we say a weak Malcev basis $X_{1}, \ldots, X_{p}$ of $\underline{\mathrm{G}}$ is subordinate to $\Gamma$ if $d_{i}=\exp \left(X_{i}\right)$ is a weak Malcev basis for $\Gamma$.

LEMMA 1.1. Let $\Gamma$ be a discrete cocompact subgroup of $G$. If $\left\{d_{1}, \ldots, d_{p}\right\}$ is a weak Malcev basis for $\Gamma$, then $\left\{X_{i}=\log \left(d_{i}\right) \mid i=1,2, \ldots, p\right\}$ forms a weak Malcev basis of $\underline{\mathbf{G}}$ subordinate to $\Gamma$.

LEMMA 1.2. Let $G$ and $\Gamma$ be as above, $M \subseteq G$ a closed connected subgroup of $G$ such that $M / M \cap \Gamma$ is compact. If $\left\{X_{1}, \ldots, X_{l}\right\}$ is a weak Malcev basis of $\underline{\mathbf{M}}$ subordinate to $\Gamma \cap M$, then it can be extended to a weak Malcev basis of $\underline{\mathbf{G}}$ subordinate to $\Gamma$.

REMARK. If $M \subseteq G$ is a connected subgroup, then $M$ is called rational if $M \cap \Gamma$ is cocompact in $M$. If $\underline{\mathbf{M}}$ is a subalgebra of $\underline{\mathbf{G}}, \underline{\mathbf{M}}$ is rational if the connected subgroup of $G$ with Lie algebra $\underline{\mathbf{M}}$ is rational. See the appendix to [C-G-1] for more details.

Finally, we need the following lemma from $[\mathbf{H}-\mathbf{1}]$ or $[\mathbf{R}]$.

LEMMA 1.3. Let $\Gamma, G$ be as above with $\underline{\mathbf{G}}$ the Lie algebra of $G$. If $\underline{\mathbf{Z}}$ is the center of $\underline{\mathbf{G}}$ we will suppose that $\operatorname{dim}(\underline{\mathbf{Z}})=1$. Let $Z \in \log (\Gamma) \cap \underline{\mathbf{Z}}$ be a generator for this lattice. Then there exist $Y \in \underline{\mathbf{G}}$ such that if $W=\operatorname{span}_{\mathbb{R}}(Z, Y)$ then $Y$ and $Z$ are generators for $\log (\Gamma) \cap W$. Let $\underline{\mathbf{G}}_{1}$ be the centralizer of $Y$ in $\underline{\mathbf{G}}$, then $\underline{\mathbf{G}}_{1}$ is rational and of codimension 1 in $\mathbf{G}$. There is $X \in \log (\Gamma)$ so that if $\Gamma_{1}=\Gamma \cap \exp \left(\underline{\mathbf{G}}_{1}\right)$, then $\Gamma_{1}$ and $\exp (X)$ generate $\Gamma$. If $L=\operatorname{span}_{\mathbb{R}}(X, Y, Z)$, then $L$ is a three dimensional Heisenberg algebra and $\exp (X), \exp (Y)$, and $\exp (Z)$ generate $\Gamma \cap \exp (L)$. Finally there exist $a \in \mathbb{Z}, a \neq 0$, so that $[X, Y]=a Z$ and $a$ is independent of the choice of $X$ satisfying the above conditions.

Let $\Gamma \subseteq G$ and $\pi=\operatorname{ind}_{H}^{G}\left(X_{f}\right)$, an irreducible representation of $G$. Suppose there exists $g \in G$ such that

(a) $\Gamma g H$ is closed in $G$; equivalently $g \mathrm{Hg}^{-1} / \mathrm{gHg}^{-1} \cap \Gamma$ is compact;

(b) $\left.\quad \chi_{\mathrm{Ad}^{*}(g) f}\right|_{g \mathrm{Hg}^{-1} \cap \Gamma} \equiv 1$.

For each $g$ we can define an element $D_{g} \in H^{-\infty}(\pi)$. For $\phi \in H^{\infty}(\pi)$ we have

$$
D_{g}(\phi)=\sum_{\gamma \in \Gamma / \Gamma \cap g H g^{-1}} \phi(\gamma \cdot g) \text {. }
$$

Condition (1.1)(b) says (1.2) is well defined if it converges. We get that it converges and defines an element in $H^{-\infty}(\pi)$ from (1.1)(a). Applying Lemma 1.2 to $g \mathrm{Hg}^{-1}$ we can find a weak Malcev basis $X_{1}, \ldots, X_{r}$ of $\operatorname{Ad}(g)(\underline{\mathbf{H}})$ subordinate to 
$g \mathrm{Hg}^{-1} \cap \Gamma$ and an extension $X_{1}, \ldots, X_{r}, X_{r+1}, \ldots, X_{n}$ to a weak Malcev of $\underline{\mathbf{G}}$ subordinate to $\Gamma$. Then

$$
S=\left\{\exp \left(t_{n} X_{n}\right) \cdots \exp \left(t_{r+1} X_{r+1}\right) \mid t_{i} \in \mathbb{R}\right\}
$$

is a cross section for $G / H$ and $\exp \left(t_{n} X_{n}\right) \cdots \exp \left(t_{r+1} X_{r+1}\right) \in \Gamma$ if and only if $t_{i}$ is an integer for $i=r+1, \ldots, n$. Writing $F(\bar{t})=\exp \left(t_{n} X_{n}\right) \cdots \exp \left(t_{r+1} X_{r+1}\right)$ for our coordinate map, we have

$$
D_{g}(\phi)=\sum_{\bar{m}=\left(m_{n}, \ldots, m_{r+1}\right) \in \mathbb{Z}^{n-r}} \phi(F(\bar{m})) .
$$

Since $\phi \circ F \in \mathscr{S}\left(\mathbb{R}^{n-r}\right)$ we have (1.3) converges and defines an element in $H^{-\infty}(\pi)$. Note that conditions (1.1)(a) and (b) depend only on the $\Gamma g H$ double coset to which $g$ belongs and if we change $g$ to $\bar{g} \in \Gamma g H$, then $D_{g}$ and $D_{\bar{g}}$ differ by a scalar of absolute value one. Also if $g_{1}, \ldots, g_{n}$ are representatives of distinct double cosets, then the supports of $D_{g_{i}}$ are mutually disjoint, so $D_{g_{i}}$ are linearly independent. Consequently, Theorem 0.1 says the multiplicity of $\pi$ is bigger than or equal to the number of double cosets satisfying (1.1). We will have the Howe-Richardson multiplicity formula if, for rational $H$, we can show all of the above distributions span the $\Gamma$-invariant elements in $H^{-\infty}(\pi)$.

Suppose $H_{1}$ and $H_{2}$ are polarizations for $f$ and set $\pi_{1}=\operatorname{ind}_{H_{1}}^{G}\left(\chi_{f}\right), \pi_{2}=$ ind $_{H_{2}}^{G}\left(\chi_{f}\right)$. Then according to the Kirillov theory, $\pi_{1}$ is equivalent to $\pi_{2}$ so there exists a unitary intertwining map $T: H\left(\pi_{1}\right) \rightarrow H\left(\pi_{2}\right)$. Formally, it is easy to write down the intertwining map $T$. For $\phi \in H\left(\pi_{1}\right)$ we define $T(\phi) \in H\left(\pi_{2}\right)$ by

$$
T(\phi)(x)=\int_{H_{2} / H_{1} \cap H_{2}} \phi(x h) \chi_{f}(h) d h .
$$

To show that (1.4) defines an intertwining operator we need the following lemma, its proof almost identical to Penney's proof of Theorem 0.1.

Lemma 1.4. Let $D \in H^{-\infty}\left(\pi_{1}\right)$ such that $\pi_{1}(h) D=\bar{\chi}(h) D$ for all $h \in H_{2}$. If $\phi \in H^{\infty}\left(\pi_{1}\right)$ defines $T_{D}(\phi)$ by $T_{D}(\phi)(g)=\left(\pi_{1}(g) D\right)(\phi)$, then $T_{D}$ defines a bounded intertwining operator from $H\left(\pi_{1}\right) \rightarrow H\left(\pi_{2}\right)$.

Proof. Define a sesquilinear pairing $\beta$ on $H^{\infty}\left(\pi_{1}\right) \times H^{\infty}\left(\pi_{2}\right)$ by

$$
\beta\left(\phi_{1}, \phi_{2}\right)=\int_{G / H_{2}}\left(\pi_{1}(g) D\right)\left(\phi_{1}\right) \bar{\phi}_{2}(g) d \bar{g} .
$$

To see that the integral in (1.5) converges we estimate $\left|\left(\pi_{1}(g) D\right)(\phi)\right|$. Since $D \in$ $H^{\infty}(\pi)$ we can find elements $u_{i} \in \underline{\mathbf{U}}(\underline{\mathbf{G}})$ and a constant $K>0$ so that

$$
|D(\phi)| \leqslant K \sum_{i=1}^{l}\left\|\pi\left(u_{i}\right) \phi\right\| .
$$

Consequently we have

$$
|\pi(g) D(\phi)|=\left|D\left(\pi\left(g^{-1}\right) \phi\right)\right| \leqslant \sum_{i=1}^{l}\left\|\pi\left(\operatorname{Ad}(g) u_{i}\right) \cdot \phi\right\| .
$$

Let $V$ be a finite dimensional $\operatorname{Ad}(g)$ invariant subspace of $\underline{\mathbf{U}}(\underline{\mathbf{G}})$ that contains $u_{1}, \ldots, u_{l_{0}}$. If $l_{1}, \ldots, l_{m}$ is a basis of $V$, there exist polynomials $a_{i j}(g)$ such that $\operatorname{Ad}(g) u_{i}=\sum_{j=1}^{m} a_{i j}(g) l_{j}$. Consequently we have that $|\pi(g) D(\phi)|$ grows no faster 
than a polynomial on $G / H_{2}$. Since $\phi_{2} \in \mathscr{S}\left(G / H_{2}\right)$ it is clear that (1.5) converges and is separately continuous in each variable.

Since $\beta\left(\pi_{1}(g) \phi_{1}, \phi_{2}\right)=\beta\left(\phi_{1}, \pi_{2}\left(g^{-1}\right) \phi_{2}\right)$, we can apply Theorem 2.1 of [Po] to conclude that there exist $T: H^{\infty}\left(\pi_{1}\right) \rightarrow H^{\infty}\left(\pi_{2}\right)$ such that $\beta\left(\phi_{1}, \phi_{2}\right)=\left\langle T \phi_{1}, \phi_{2}\right\rangle$, where $\langle$,$\rangle is the inner product on H\left(\pi_{2}\right)$. Now Schur's lemma implies that $T$ extends to a bounded operator from $H\left(\pi_{1}\right)$ to $H\left(\pi_{2}\right)$. If $\phi_{1} \in H^{\infty}(\pi)$ and we let $\phi_{2}^{\alpha}$ run through an $L^{1}$-approximate identity we see that $T\left(\phi_{1}\right)(g)=T_{D}(\phi)(g)$, so $T_{D}$ does define a bounded intertwining operator from $H\left(\pi_{1}\right) \rightarrow H\left(\pi_{2}\right)$. Q.E.D.

LEMMA 1.5. For $\phi \in H^{\infty}\left(\pi_{1}\right)$ define

$$
D(\phi)=\int_{H_{2} / H_{1} \cap H_{2}} \phi(h) \chi_{f}(h) d h .
$$

Then $D \in H^{-\infty}\left(\pi_{1}\right)$ and $\pi_{1}(h) D=\chi_{f}\left(h^{-1}\right) D$ for all $h \in H_{2}$.

Proof. The second assertion is clear and the first follows from the identification of $H^{\infty}\left(\pi_{1}\right)$ as $\mathscr{S}\left(G / H_{1}\right)$. Q.E.D.

If follows from Lemmas 1.4 and 1.5 that (1.4) holds for all $\phi \in H^{\infty}\left(\pi_{1}\right)$ and extends to a bounded intertwining map from $H\left(\pi_{1}\right)$ to $H\left(\pi_{2}\right)$.

Let $f \in \underline{\mathbf{G}}^{*}, H$ a polarization for $f$, and suppose there exists $g \in G$ so that conditions (1.1)(a) and (b) hold. By relabeling $f$ and $H$ we can assume that $\Gamma H$ is closed in $G$ and $\left.\chi_{f}\right|_{H \cap \Gamma} \equiv 1$. Now this second condition implies that if $\bar{f}$ is the restriction of $f$ to $\underline{\mathbf{H}}$ then $\bar{f} \in \underline{\mathbf{H}}_{Q}^{*}$ so, by changing $f$ by an element of $\underline{\mathbf{H}}^{\perp}$ if necessary, we can assume that $f \in \underline{\mathbf{G}}_{Q}$. (Recall that $f+\underline{\mathbf{H}}^{\perp}=\operatorname{Ad}^{*}(H) \cdot f$, so $H$ stays a polarization for $f$ and the representation $\pi_{1}$ does not change at all.) The first condition says that $H$ is a rational subgroup of $G$.

If $H$ is a rational polarization for the rational functional $f$, we denote by $A(H)$ the C-linear span of the elements of $H^{-\infty}\left(\operatorname{ind}_{H}^{G}\left(\chi_{f}\right)\right)$ constructed in (1.2).

LEMmA 1.6. Let $S=\left\{g_{1}, \ldots, g_{l}\right\}$ be a set of double coset representatives for $\Gamma: H$ such that $\Gamma \mathrm{gH}$ is a closed coset and $\left.\chi_{\mathrm{Ad}^{*}(g)} f\right|_{g_{g^{-1}} \cap \Gamma} \equiv 1$. Then we can choose $g_{1}, \ldots, g_{\text {l }}$ to be rational elements of $G$.

Proof. Since $\Gamma g H$ is closed, $\Gamma \cap g \mathrm{Hg}^{-1}$ is cocompact in $\mathrm{gHg}^{-1}$; hence $\mathrm{gHg}^{-1}$ is a rational subgroup of $G$ with rational Lie algebras $\operatorname{Ad}(g)(\underline{\mathbf{H}})$. As before, we restrict $\operatorname{Ad}^{*}(g)(f)$ to $\operatorname{Ad}(g)(\underline{\mathbf{H}})$. Then we get a rational element in $\operatorname{Ad}(g)(\underline{\mathbf{H}})$. By changing $g$ on the right by an element of $H$ we can assume that $\operatorname{Ad}^{*}(g)(f)$ is a rational element of $\underline{G}^{*}$. Now it follows from the theory of algebraic groups that we can find a rational $\bar{g} \in G$ such that $\operatorname{Ad}^{*}(\bar{g}) f=\operatorname{Ad}^{*}(g) f$. Since $\bar{g}$ and $g$ must differ by an element of the stabilizer of $f$ which is contained in $H, \bar{g}$ and $g$ define the same $\Gamma$ : $H$ double coset. This proves the lemma. Q.E.D.

LEMMA 1.7. Let $f \in \mathbf{G}^{*}$ be a rational element and $H_{1}, H_{2}$ two rational polarizations for $f$. Let $\pi_{1}=\operatorname{ind}_{H_{1}}^{G}\left(\chi_{f}\right), \pi_{2}=\operatorname{ind}_{H_{2}}^{G}\left(\chi_{f}\right)$ and $T: H\left(\pi_{1}\right) \rightarrow H\left(\pi_{2}\right)$ the intertwining operator of (1.4). Let $T$ also denote the restriction of $T$ to the space of $C^{\infty}$-vectors and $T^{*}: H^{-\infty}\left(\pi_{2}\right) \rightarrow H^{-\infty}\left(\pi_{1}\right)$ the dual map. Then we have $T^{*}\left(A\left(H_{2}\right)\right)=T^{*}\left(A\left(H_{1}\right)\right)$. 
Proof. Since $T^{*}$ is invertible it is enough to show $T^{*}\left(A\left(H_{2}\right)\right) \subseteq A\left(H_{1}\right)$. This is trivial if $A\left(\mathrm{H}_{2}\right)$ is the zero subspace, so we may as well assume $A\left(\mathrm{H}_{2}\right)$ is nontrivial. We use induction on the dimension of $\underline{\mathbf{G}}$ and, as usual, reduce to the case where $\operatorname{dim}(\operatorname{ker}(f) \cap \operatorname{center}(\underline{\mathbf{G}}))=0$. Let $X, Y, Z$, and $\underline{\mathbf{G}}_{1}$ be as in Lemma 1.3, so $[X, Y]=a Z, f(Z)=\lambda$, and $a$ and $\lambda$ are both integers. Let $G_{1}=\exp \left(\underline{G}_{1}\right)$. Then we have $G=G_{1} \cdot\{\exp (t X) \mid t \in \mathbb{R}\}$. We will suppose that $H_{1}$ and $H_{2}$ are subgroups of $G_{1}$.

For any $g \in G$ we can write $g=r \cdot s$ with $r \in G_{1}$ and $s=\exp (t X)$. In particular, if $g$ is a rational element of $G$ then $r$ will be a rational element of $G_{1}$ and $s$ will be a rational element of $G$. Of course we have the same sort of splitting for $\gamma \in \Gamma$. We can write $\gamma=\exp (n X) \cdot \gamma_{1}$ where $n \in \mathbb{Z}$ and $\gamma_{1} \in \Gamma_{1}=\Gamma \cap G_{1}$. Now pick an element $D_{g} \in A\left(H_{2}\right)$. Then by Lemma 1.6 we saw that we may assume that $g$ is a rational element of $G$. Let $g=r \cdot s$ be as above and set $M=H_{2} / H_{1} \cap H_{2}$. We will write $M(s)$ for $s H_{2} s^{-1} / s\left(H_{1} \cap H_{2}\right) s^{-1}$, and we will write $\bar{f}$ for $\mathrm{Ad}^{*}(s) f$. Finally we will let $C$ denote a set of coset representatives for $\Gamma_{1} \cap g H_{2} g^{-1}$ in $\Gamma_{1}$. For $\phi \in H^{\infty}\left(\pi_{1}\right)$ we have

$$
\begin{aligned}
T^{*}\left(D_{g}\right)(\phi) & =D_{g}(T(\phi))=\sum_{\gamma \in \Gamma / \Gamma \cap g H_{2} g^{-1}} \int_{M} \phi(\gamma g h) \chi_{f}(h) d h \\
& =\sum_{n \in \mathbf{Z}} \sum_{\gamma_{1} \in C} \int_{M} \phi\left(\exp (n X) \gamma_{1} r s \cdot h \cdot s^{-1} \cdot s\right) \chi_{f}(h) d h \\
& =\sum_{n \in \mathbf{Z}} \sum_{\gamma_{1} \in C} \int_{M(s)} \phi\left(\exp (n X) \gamma_{1} r h \cdot s\right) \chi_{\bar{f}}(h) d h .
\end{aligned}
$$

Now suppose $\phi$ is of the form $\phi\left(\exp (t X) \cdot g_{1}\right)=\alpha(t) \cdot \beta\left(g_{1}\right)$ with $\alpha \in \mathscr{S}(\mathbb{R})$ and $\beta \in H^{\infty}\left(\operatorname{ind}_{H_{1}}^{G_{1}}\left(\chi_{f}\right)\right)$. The span of such $\phi$ 's is dense in $H^{\infty}\left(\pi_{1}\right)$. Now we compute the integral of (1.8) when $\phi$ is of this special form. Recalling that $s=\exp (t X)$ we have

$$
\exp (n X) \gamma_{1} r h s=\exp (n X) s \cdot s^{-1} \gamma_{1} r h s=\exp ((n+t) X) \cdot s^{-1} \gamma_{1} r h s
$$

and $s^{-1} \gamma_{1} r h s \in G_{1}$. If we set $\bar{\beta}\left(g_{1}\right)=\beta\left(s^{-1} g_{1} s\right)$ we have that (1.8) can be written as

$$
\begin{aligned}
T^{*}\left(D_{g}\right)(\phi) & =\sum_{n \in \mathbb{Z}} \sum_{\gamma_{1} \in C} \alpha(n+t) \int_{M(s)} \bar{\beta}\left(\gamma_{1} r h\right) \chi_{\bar{f}}(h) d h \\
& =\sum_{n \in \mathbb{Z}} \alpha(n+t) \sum_{\gamma_{1} \in C} \int_{M(s)} \bar{\beta}\left(\gamma_{1} r h\right) \chi_{\bar{f}}(h) d h .
\end{aligned}
$$

Now we note that $\bar{\beta}$ is an element of $H^{\infty}\left(\operatorname{ind}_{s H_{2} s^{-1}}^{G_{1}}\left(\chi_{\bar{f}}\right)\right)$. Let $T^{1}$ be the intertwining operator from ind ${ }_{s H_{2} s^{-1}}^{G_{1}}\left(\chi_{\bar{f}}\right)$ to $\operatorname{ind}_{s H_{1} s^{-1}}^{G_{1}}\left(\chi_{\bar{f}}\right)$ given by (1.4). Let $D_{r}^{1} \in$ $H^{-\infty}\left(\operatorname{ind}_{s H_{2} s^{-1}}^{G_{1}}\left(\chi_{\bar{f}}\right)\right)$ be given by $D_{r}^{1}(\bar{\beta})=\sum_{\gamma_{1} \in \Gamma_{1} / \Gamma_{1} \cap s H_{2} s^{-1}} \bar{\beta}\left(\gamma_{1} \cdot r\right)$. Then we have that (1.9) is equal to

$$
\sum_{n \in \mathbf{Z}} \alpha(n+t)\left(T^{1}\right)^{*}\left(D_{r}^{1}\right)(\bar{\beta}) .
$$

Now we can apply our induction hypothesis to get rational elements $g_{1}, \ldots, g_{l} \in G_{1}$ and constants $c_{1}, \ldots, c_{l}$ in $\mathbf{C}$ so that

(i) $\Gamma_{1} g_{i} s H_{1} s^{-1}$ is closed in $G_{1}$;

(ii) $\left.\chi_{\operatorname{Ad}^{*}\left(g_{i}\right) \hat{f}}\right|_{\Gamma_{1} \cap g_{i} s H s^{-1} g_{i}^{-1}} \equiv 1$;

(iii) $\left(T^{1}\right)^{*}\left(D_{R}^{1}\right)=\sum_{i=1}^{l} c_{i} D_{g_{i}}^{1}$. 
Now we just work backward to compute (1.10). Let $S_{i}$ be a set of coset representatives for $\Gamma_{1} \cap g_{i} s H_{1} s^{-1} g_{i}^{-1}$ in $\Gamma_{1}$. Then we have that (1.10) is equal to

$$
\sum_{i=1}^{l} c_{i} \sum_{n \in \mathbb{Z}} \alpha(n+t) \sum_{\gamma_{1} \in S_{i}} \bar{\beta}\left(\gamma \cdot g_{i}\right)=\sum_{i=1}^{l} c_{i} \sum_{\gamma \in \Gamma / \Gamma \cap g_{i} s H s^{-1} g_{i}^{-1}} \psi\left(\gamma g_{i} \cdot s\right) .
$$

This is a sum of elements in $A\left(H_{1}\right)$.

Now we will show how to reduce to the case just considered. Therefore we consider a rational polarization $H$ for $f$ such that $\left.\chi_{f}\right|_{H \cap \Gamma} \equiv 1$. As in the usual argument, we form the new polarization $H_{2}=\left(H \cap G_{1}\right) \cdot\{\exp (t Y) \mid t \in \mathbb{R}\}$. This $H_{2}$ is rational since $H \cap G_{1}$ is rational and $\{\exp (t Y) \mid t \in \mathbb{R}\}$ is a rational subgroup and clearly contained in $G_{1}$. Let $\pi_{1}=\operatorname{ind}_{H}^{G}\left(\chi_{f}\right), \pi_{2}=\operatorname{ind}_{H_{2}}^{G}\left(\chi_{f}\right)$, and $T: H\left(\pi_{1}\right) \rightarrow$ $H\left(\pi_{2}\right)$ the intertwining operator given in (1.4). We wish to show that $T^{*}\left(A\left(H_{2}\right)\right) \subseteq$ $A\left(H_{1}\right)$. Thus let $D_{g} \in A\left(H_{2}\right)$, with $g$ rational. If $X$ is any vector in $\underline{\mathbf{H}}$ that complements $\underline{\mathbf{G}}_{1}$ we have that $g=r \circ s$ with $s=\exp (t X)$ for suitable $t$. If we pick a rational vector in $\underline{\mathbf{H}}$ to complement $\underline{\mathbf{G}}_{1}$, then we can assume that $s=\exp (X)$. Now $r\left(H \cap G_{1}\right) r^{-1} \subseteq r H r^{-1}$ is a sequence of rational subgroups, so by Lemma 1.2 there exists $\bar{X} \in \operatorname{Ad}(r)(\underline{\mathbf{H}})$ so that

$$
\Gamma \cap r H r^{-1}=\{\exp (n \bar{X}) \mid n \in \mathbb{Z}\} \cdot\left(\Gamma \cap r\left(H \cap G_{1}\right) r^{-1}\right) .
$$

If we set $\bar{\Gamma}=\{\exp (n \bar{X}) \mid n \in \mathbb{Z}\} \cdot \Gamma_{1}$, then $\bar{\Gamma}$ is of finite index in $\Gamma$. Let $\delta_{1}, \ldots, \delta_{L}$ be a set of coset representatives for $\bar{\Gamma}$ in $\Gamma$. Thus for $\gamma \in \Gamma$ we have that there exists $m \in \mathbb{Z}, 1 \leqslant i \leqslant L$, and $\gamma_{1} \in \Gamma_{1}$ so that $\gamma=\delta_{i} \gamma_{1} \cdot \exp (m \bar{X})$. We will let $S$ be a set of coset representatives for $\Gamma_{1} \cap g H_{2} g^{-1}$ in $\Gamma_{1}$. Finally set $\mu=f(Y)$ and note that the assumption $\left.\chi_{\mathrm{Ad}^{*}(g)(f)}\right|_{\Gamma \cap g H_{2} g^{-1}} \equiv 1$ says that

$$
\operatorname{Ad}^{*}(g)(f)(Y)=\operatorname{Ad}^{*}(\exp (X)) f(Y)=f(Y+c Z)=\mu+c \lambda
$$

is an integer. (Here we have $[X, Y]=c Z$, but since we have changed the $X$ so that it lies in $\underline{\mathbf{H}}$ we can only conclude that $c$ is a rational number.)

Let $\phi \in H^{\infty}\left(\pi_{1}\right)$. Then we have

$$
\begin{gathered}
T^{*}\left(D_{g}\right)(\phi)=\sum_{\gamma \in \Gamma / \Gamma \cap g H_{2} g^{-1}} \int_{-\infty}^{\infty} \phi(\gamma g \exp (\tau Y)) \exp (2 \pi i \mu \tau) d \tau \\
=\sum_{m \in \mathbf{Z}} \sum_{\gamma_{1} \in S} \sum_{i=1}^{L} \int_{-\infty}^{\infty} \phi\left(\delta_{i} \gamma_{1} \exp (m \bar{X}) \cdot r \cdot s \exp (\tau Y)\right) \exp (2 \pi i \mu \tau) d \tau .
\end{gathered}
$$

Now we note that

$$
\begin{aligned}
\delta_{i} \gamma_{1} \exp (m \bar{X}) r \cdot s \cdot & \exp (\tau Y) \\
& =\delta_{i} \gamma_{1} r \exp (\tau Y) \cdot \exp \left(m \operatorname{Ad}\left(r^{-1}\right)(\bar{X})\right) \cdot \exp (\tau(c+b m) Z)
\end{aligned}
$$

where $[X, Y]=c Z$ as above and $[\bar{X}, Y]=b Z$ where $b$ is an integer. Now $\exp \left(m\left(\operatorname{Ad}\left(r^{-1}\right) \bar{X}\right)\right), \exp (X)$, and $\exp (\tau(c+b m) Z)$ are all in $H$ and $\phi$ is a function such that $\phi(g h)=\bar{\chi}_{f}(h) \phi(g)$ for all $h \in H$. Therefore we get

$$
\begin{aligned}
\phi\left(\delta_{i} \gamma_{1} \exp (m \bar{X}) r \cdot s \cdot \exp (\tau Y)\right)=\phi\left(\delta_{i} \gamma_{1} r \cdot \exp (\tau Y)\right) \\
\cdot \exp \left(-2 \pi i\left(m f\left(\operatorname{Ad}\left(r^{-1}\right)(\bar{X})\right)+f(X)+\lambda \tau(c+b m)\right)\right) .
\end{aligned}
$$


Therefore for $m, i$, and $\gamma_{1}$ fixed, we have that the integrand in (1.13) is

$$
\phi\left(\delta_{i} \gamma_{1} r \exp (\tau Y)\right) \circ \exp \left(-2 \pi i\left(m f\left(\operatorname{Ad}\left(r^{-1}\right)(\bar{X})\right)+f(X)+\lambda \tau(\bar{c}+b m)\right)\right)
$$

where $\bar{c}=c+\mu / \lambda$.

If in the integrand we make the change of variables $\tau \rightarrow \tau+d,(1.15)$ turns into

$$
\phi\left(\delta_{i} \gamma_{1} r \exp (\tau+d)(Y)\right) \exp ((\tau+d)(Y)) \cdot L
$$

where

$$
\begin{aligned}
L= & \exp (-2 \pi i(f(X))) \\
& \cdot \exp \left(-2 \pi i\left(m f\left(\operatorname{Ad}\left(r^{-1}\right)(\bar{X})+\lambda d(\bar{c}+b m)+\lambda \tau(\bar{c}+b m)\right)\right) .\right.
\end{aligned}
$$

Regrouping the term in the exponent of the exponential factor we get that $(1.16)$ is equal to

$$
K \cdot \phi\left(\delta_{i} \gamma_{1} r \exp ((\tau+d)(Y))\right) \exp \left(-2 \pi i\left(m\left(f\left(\operatorname{Ad}\left(r^{-1}\right)(\bar{X})\right)+\lambda \tau(\bar{c}+b m)\right)\right)\right)
$$

where $K=\exp (-2 \pi i(f(X)+\lambda d \bar{c}))$ is a nonzero constant. If we choose $d$ so that $f\left(\operatorname{Ad}\left(r^{-1}\right)(\bar{X})\right)+\lambda d b=0,(1.17)$ becomes

$$
K \cdot \phi\left(\delta_{i} \gamma_{1} r \cdot \exp (d Y) \cdot \exp (\tau Y)\right) \cdot \exp (-2 \pi i(\lambda \tau(\bar{c}+b m)))
$$

Hence we can write (1.13) as

$$
\begin{array}{r}
T^{*}\left(D_{g}\right)(\phi)=K \sum_{\gamma \in S_{1}} \sum_{i=1}^{1} \sum_{m \in \mathbb{Z}} \int_{-\infty}^{\infty} \phi\left(\delta_{i} \gamma_{1} r \cdot \exp (d Y) \cdot \exp (\tau Y)\right) \\
\cdot \exp (-2 \pi i(\lambda \tau(\bar{c}+b m))) d t
\end{array}
$$

To simplify (1.19) we will use the Poisson summation formula on

$$
\sum_{m \in \mathbb{Z}} \int_{-\infty}^{\infty} \phi\left(\delta_{i} \gamma_{1} r \exp (d Y) \cdot \exp (\tau Y)\right) \exp (-2 \pi i \tau((\mu+c \lambda)+\lambda b m)) .
$$

Recall the classical Poisson summation formula on $\mathbb{R}$. If $F \in \mathscr{S}(\mathbb{R})$ we have

$$
\hat{F}(\xi)=\int_{-\infty}^{\infty} F(x) \exp (-2 \pi i \xi \chi) d x
$$

and $\hat{F} \in \mathscr{S}(\mathbb{R})$. If $\alpha$ and $\beta$ are any integers we have

$$
\sum_{n \in \mathbb{Z}} \hat{F}(n \beta+\alpha)=\frac{1}{\beta} \sum_{n \in \mathbb{Z}} F\left(\frac{n}{\beta}\right) \cdot \exp \left(2 \pi\left(\frac{n}{\beta}\right) \alpha\right) .
$$

Let $F(\tau)=\phi\left(\delta_{i} \gamma_{1} r \exp (d Y) \cdot \exp (\tau Y)\right)$ and set $\alpha=\mu+c \lambda$ and $\beta=\lambda b$ (recall that both $\alpha$ and $\beta$ are integers). Then we have

$\int_{-\infty}^{\infty} \phi\left(\delta_{i} \gamma_{1} r \exp (d Y) \exp (\tau(Y)) \exp (-2 \pi i \tau((\mu+c \lambda)+\lambda b m))\right) d \tau=\hat{F}(\beta m+\alpha)$.

Consequently (1.20) can be written, using (1.21), as

$$
\sum_{n \in \mathbb{Z}} \hat{F}(\beta m+\alpha)=\frac{1}{\beta} \sum_{m \in \mathbb{Z}} \exp \left(2 \pi i\left(\frac{m}{\beta}\right) \alpha\right) \cdot \phi\left(\delta_{i} \gamma_{1} r \exp (d Y) \exp \left(\frac{m}{\beta} Y\right)\right) .
$$


If we write $m=\beta n+k$ with $0 \leqslant k \leqslant \beta-1, k$ an integer, then (1.22) becomes

$$
\frac{1}{\beta} \sum_{k=0}^{\beta-1} \exp \left(2 \pi i\left(\frac{k}{\beta}\right) \alpha\right) \sum_{n \in \mathbb{Z}} \phi\left(\delta_{i} \gamma_{1} \exp (n Y) \cdot r \cdot \exp \left(d+\frac{k}{\beta}\right)(Y)\right) \text {. }
$$

Set $g_{k}=r \cdot \exp ((d+k / \beta)(Y))$ for $0 \leqslant k \leqslant \beta-1$. Putting this together yields

$$
T^{*}\left(D_{g}\right)(\phi)=\frac{K}{\beta} \sum_{k=0}^{\beta-1} \exp \left(2 \pi i\left(\frac{k \alpha}{\beta}\right)\right) \cdot \sum_{i=1}^{L} \sum_{m \in \mathbb{Z}} \sum_{\gamma_{1} \in S} \phi\left(\delta_{i} \gamma_{1} \exp (m Y) \cdot g_{k}\right) .
$$

Finally, noting that $\left\{\delta_{i} \gamma_{1} \exp (m Y) \mid 1 \leqslant i \leqslant L, \gamma_{1} \in S, m \in \mathbb{Z}\right\}$ forms a set of coset representatives for $g_{k} H g_{k}^{-1} \cap \Gamma$ in $\Gamma$, we conclude that $T^{*}\left(D_{g}\right) \in A(H)$. To summarize, we have shown that if $H$ is a rational polarization for $f$ but $H$ is not contained in $G_{1}$, then we can construct $H_{2}=\left(H \cap G_{1}\right) \cdot \exp (\mathbb{R} Y)$, a rational polarization for $f$ with $H_{2}$ contained in $G_{1}$. In addition if $\pi_{1}=\operatorname{ind}_{H_{1}}^{G}\left(\chi_{f}\right), \pi_{2}=$ ind $_{H_{1}}^{G}\left(\chi_{f}\right)$, and $T: H\left(\pi_{1}\right) \rightarrow H\left(\pi_{2}\right)$ is the intertwining operator of (1.4), then $T^{*}\left(A\left(H_{2}\right)\right)=A\left(H_{1}\right)$.

Now suppose $H$ and $B$ are two rational polarizations for $f$ and assume that neither $H$ nor $B$ is contained in $G_{1}$. Then we can form $H_{2}=\left(H \cap G_{1}\right) \cdot \exp (\mathbb{R} Y)$ and $B_{2}=\left(B \cap G_{1}\right) \cdot \exp (\mathbb{R} Y)$ and the corresponding representations $\pi_{1}=$ $\operatorname{ind}_{H}^{G}\left(\chi_{f}\right), \pi_{2}=\operatorname{ind}_{H_{2}}^{G}\left(\chi_{f}\right), \sigma_{1}=\operatorname{ind}_{B}^{G}\left(\chi_{f}\right)$ and $\sigma_{2}=\operatorname{ind}_{B_{2}}^{G}\left(\chi_{f}\right)$. We consider the following, where all intertwining operators are given by (1.4).

$$
\begin{array}{ccc}
H^{\infty}\left(\pi_{1}\right) & \stackrel{T_{1}}{\rightarrow} & H^{\infty}\left(\sigma_{1}\right) \\
T_{4} \downarrow & & \downarrow T_{2} \\
H_{2}^{\infty}(\pi) & \vec{T}_{3} & H^{\infty}\left(\sigma_{2}\right)
\end{array}
$$

By Schur's lemma $T_{1}=C \cdot\left(T_{2}\right)^{-1} \cdot T_{3} \cdot\left(T_{4}\right)$ for some constant $C$. Consequently we can normalize $T_{1}$ so that $T_{1}=\left(T_{2}\right)^{-1} \cdot T_{3} \cdot T_{4}$. Recalling that $A(H) \subseteq H^{-\infty}\left(\pi_{1}\right)$, $A\left(H_{2}\right) \subseteq H^{-\infty}\left(\pi_{2}\right), A(B) \subseteq H^{-\infty}\left(\sigma_{1}\right)$, and $A\left(B_{2}\right) \subseteq H^{-\infty}\left(\sigma_{2}\right)$ we have

$$
\begin{array}{ccc}
A(H) & & A(B) \\
T_{4}^{*} \uparrow & & \uparrow T_{2}{ }^{*} \\
A\left(H_{2}\right) & \leftarrow & A\left(B_{2}\right)
\end{array}
$$

Consequently $\left(T_{1}\right)^{*}(A(B))=A(H)$.

Thus we see that $H_{1}, H_{2}$ are two rational polarizations for $f$ and $\pi_{1}=\operatorname{ind}_{H_{1}}^{G}\left(\chi_{f}\right)$, $\pi_{2}=\operatorname{ind}_{H_{2}}^{G}\left(\chi_{f}\right)$ with corresponding set of $\Gamma$ invariants $A\left(H_{1}\right), A\left(H_{2}\right)$. Then $\left(T^{*}\right)\left(A\left(H_{2}\right)\right)=A\left(H_{1}\right)$. Q.E.D.

LEMMA 1.8. Let $f \in \underline{\mathbf{G}}^{*}$ be a rational element and $H$ a rational polarization for $f$. Let $\pi=\operatorname{ind}_{H}^{G}\left(\chi_{f}\right)$ and $A(H) \subseteq H^{-\infty}(\pi)$, the subspace of $\Gamma$ invariant distributions constructed in (1.2). Suppose $D \in H^{-\infty}(\pi)$ such that $\pi(\gamma) D=D$ for all $\gamma \in \Gamma$, then $D \in A(H)$.

Proof. Using induction on the dimension of $\underline{\mathbf{G}}$ we reduce to the case where the dimension of the center of $\underline{\mathbf{G}}$ is one and $f$ is nontrivial on the center. Let $X, Y, Z$, and $\underline{G}_{1}$ be as in Lemma 1.3. Using Lemma 1.7 we can assume that $H$ is contained in 
$G_{1}$. Let $\tau=\operatorname{ind}_{H}^{G_{1}}\left(\chi_{f}\right)$, so $\pi=\operatorname{ind}_{G_{1}}^{G}(\tau)$. If we identify $G / G_{1}$ with $\mathbb{R}$ via the cross section $G / G_{1} \simeq\{\exp (t X) \mid t \in \mathbb{R}\}$, then we can identify $H^{\infty}(\pi) \simeq \mathscr{S}(\mathbb{R}) \hat{\otimes} H^{\infty}(\tau)$, where we have taken the projective tensor product. We can also identify $H^{\infty}(\tau)$ with $\mathscr{S}\left(\mathbb{R}^{l}\right)$ for some $l$, so that

$$
H^{\infty}(\pi) \simeq \mathscr{S}(\mathbb{R}) \hat{\otimes} \mathscr{S}\left(\mathbb{R}^{l}\right) \simeq \mathscr{S}\left(\mathbb{R}^{l+1}\right) .
$$

Under this identification, if $\phi \in H^{\infty}(\pi)$ and $\mu=f(Y)$ then

$$
(\pi(\exp (s Y)) \cdot \phi)(t, x)=\exp (2 \pi i(s \lambda a t+\mu)) \phi(t, x) .
$$

Now we need two well-known results from distribution theory.

(a) If $f \in C^{\infty}\left(\mathbb{R}^{n}\right)$ with all derivatives bounded and $D \in$ $\mathscr{S}^{*}\left(\mathbb{R}^{n}\right)$ and if $f \cdot D=0$, then $\operatorname{supp}(D) \subseteq f^{-1}(0)$.

(b) If $x \in f^{-1}(0)$ and $\nabla f(x) \neq 0$, then there exist a neighborhood $U$ of $x$ such that $\nabla f \neq 0$ in $U$ (so $f^{-1}(0) \cap U$ is a smooth manifold) and a distribution $D_{0}$ on $f^{-1}(0) \cap U$ such that if $g \in C_{c}^{\infty}(U)$ then $D(g)=D_{0}\left(\left.g\right|_{f^{-1}(0)}\right)$. In particular if $\nabla f$ never vanishes on $f^{-1}(0)$ then $D(g)=D_{0}\left(\left.g\right|_{f^{-1}(0)}\right)$ for all $g \in \mathscr{S}\left(\mathbb{R}^{n}\right)$.

Let $D$ be a $\Gamma$ invariant element in $H^{-\infty}(\pi)$ and recall that $\exp (Y) \in \Gamma$. If we set $f(t)=\exp (2 \pi i(\lambda a t+\mu))-1$, then $\Gamma$ invariance of $D$ implies $f \cdot D=0$. Consequently (1.27) (a) implies that

$$
\operatorname{supp}(D) \subseteq f^{-1}(0)=\bigcup_{k=1}^{|\lambda a|}\left(\bigcup_{n \in \mathbb{Z}}\left(\frac{k}{|\lambda a|}+n-\frac{\mu}{|\lambda a|}\right) \times \mathbb{R}^{l}\right) .
$$

Since $\nabla f \neq 0$ at any point of $f^{-1}(0)(1.27)(\mathrm{b})$ implies there exist a distribution $D_{0}$ on $f^{-1}(0)$ such that $D(g)=D_{0}\left(\left.g\right|_{f^{-1}(0)}\right)$. Thus we see that for each $n \in \mathbb{Z}, 0<k \leqslant|\lambda a|$, there exist a distribution $T_{k, n} \in \mathscr{S}^{*}\left(\mathbb{R}^{\prime}\right)$ on the hyperplane $k /|\lambda a|+n-\mu /|\lambda a| \times$ $\mathbb{R}^{l}$ such that $D=\sum_{k=1}^{|\lambda a|}\left(\sum_{n \in \mathbf{Z}} T_{k, n}\right)$.

Using invariance of $D$ under $\pi(\exp (n X))$, we see that $\pi(\exp (n X)) T_{k, 0}=T_{k, n}$.

If we let $s=\mu / \lambda a$ we have $\operatorname{Ad}^{*}(\exp (s X))(f)(Y)=0$, so if $a=\exp (s X)$ we see that each $T_{k, 0}$ defines a $\Gamma \cap G_{1}$ invariant element in $H^{-\infty}\left(\operatorname{ind}_{a a^{-1}}^{G_{1}}\left(\chi_{\operatorname{Ad}^{*}(a)(f)}\right)\right)$. Now we can apply the induction hypothesis to each $T_{k, 0}$ to conclude that $T_{k, 0} \in$ $A^{1}\left(a H a^{-1}\right) \subseteq H^{-\infty}(\tau)$. Now it follows that $D \in A(H) \subseteq H^{-\infty}(\pi)$. Consequently we have shown that if $f \in \underline{\mathrm{G}}^{*}$ is rational and $H$ is a rational polarization then $A(H)$ consist of all the $\Gamma$ invariant elements in $H^{-\infty}(\pi)$. Q.E.D.

LEMMA 1.9. Let $\pi$ be an irreducible representation of $G$ and suppose there exists a nonzero $\Gamma$ invariant element in $H^{-\infty}(\pi)$. Then there exist a rational $f \in \underline{\mathbf{G}}^{*}$ and $a$ rational polarization $H$ for $f$ such that $\pi=\operatorname{ind}_{H}^{G}\left(\chi_{f}\right)$.

Proof. Let $C$ be the center of $G$. Then $\left.\pi\right|_{c}(z)=\chi_{\pi}(z) \cdot 1$, where $\chi_{\pi}$ is a character of $C$. Let $\rho=\operatorname{ind}_{\Gamma}^{G}(1)$. Then for any $\gamma \in C \cap \Gamma$ we have $\rho(\gamma)=$ Id. Consequently if $\pi$ occurs in ind ${ }_{\Gamma}^{G}(1), \chi_{\pi}(\gamma)=1$ for all $\gamma \in C \cap \Gamma$. Consequently if 
$\operatorname{dim}\left(\operatorname{ker}\left(\chi_{\pi}\right)\right)>1$, we can divide out by a rational central subgroup and use induction on dimension of $G$. Thus we can reduce to the case where $\operatorname{dim}(C)=1$ and $\chi_{\pi}$ is locally faithful on $C$. Let $X, Y, Z$, and $\underline{\mathbf{G}}_{1}$ be as in Lemma 1.3 and $G_{1}=\exp \left(\underline{\mathbf{G}}_{1}\right)$. Then $\pi=\operatorname{ind}_{G_{1}}^{G}(\tau)$. If we view $H^{\infty}(\pi)$ as $\mathscr{S}(\mathbb{R}) \hat{\otimes} H^{\infty}(\tau)$ then we saw in Lemma 1.8 that we could choose $\tau$ so that for $\phi \in H^{\infty}(\pi)$,

$$
\pi(\exp (s Y))(\phi(t))=\exp (2 \pi i s \lambda a t) \cdot \phi(t) .
$$

With this choice of $\tau$ we had that $T_{k, 0}$ was a $\Gamma_{1}$ invariant element in $H^{-\infty}(\tau)$ and if $D$ is nonzero then $T_{k, 0}$ has to be nonzero for some $k$. Applying the induction hypothesis we get a rational $H \subseteq G_{1}$ and a rational $f \in \underline{\mathbf{G}}_{1}$ such that $\tau=\operatorname{ind}_{H}^{G_{1}}\left(\chi_{f}\right)$. Inducing in stages then says $\pi=\operatorname{ind}_{H}^{G}\left(\chi_{f}\right)$. Q.E.D.

Lemma 1.9 completes the proof of the Howe-Richardson multiplicity formula.

THEOREM 1.1. Let $G$ be a connected simply connected nilpotent Lie group and $\Gamma \subseteq G$ a discrete cocompact subgroup. Let $\pi$ be an irreducible representation of $G$. Then $\operatorname{Hom}_{\Gamma}\left(1, \pi^{-\infty}\right)$ is nonzero if and only if there exist a rational $f \in \underline{\mathbf{G}}^{*}$ and a rational polarization $H$ for $f$ such that

(a) $\pi \approx \operatorname{ind}_{H}^{G}\left(\chi_{f}\right)$

(b) there exists $g \in G$ such that $\Gamma g H$ is closed in $G$ and $\left.\chi_{\mathrm{Ad}^{*}(g)(f)}\right|_{\Gamma \cap g g^{-1}} \equiv 1$.

In this case $\operatorname{dim}\left(\operatorname{Hom}_{\Gamma}\left(1, \pi^{-\infty}\right)\right)$ is equal to the number of distinct $\Gamma: H$ double cosets such that condition (b) is satisfied.

Now one can generalize the above theorem slightly and in particular the lift maps of Richardson. We briefly indicate how to do it. Let $M$ be a closed connected subgroup of $G$ such that $\Gamma \cap M=\Delta$ is compàct in $M$. Let $\sigma$ be an irreducible representation of $M$ such that $\pi=\operatorname{ind}_{M}^{G}(\sigma)$ is irreducible. We want to describe $\operatorname{Hom}_{\Gamma}\left(1, H^{-\infty}(\pi)\right)$ in terms of $\operatorname{Hom}_{\Delta}\left(1, H^{-\infty}(\sigma)\right)$.

Recall that if $M \subseteq G$ is a connected subgroup then the notion of a rapidly decreasing function on $G / M$ is well defined, which we denote by $\mathscr{S}(G / M)$. If $\pi=\operatorname{ind}_{M}^{G}(\sigma)$ is irreducible then $H^{\infty}(\pi)$ is isomorphic to $\mathscr{S}(G / M) \hat{\otimes} H^{\infty}(\sigma)$ (projective tensor product) $[\mathbf{P}-\mathbf{4}]$.

Given $g \in G$, let $\sigma^{g}$ be the representation of $g M g^{-1}$ given by $\sigma^{g}(x)=\sigma\left(g^{-1} x g\right)$ for $x \in g \mathrm{Mg}^{-1}$. If $\Gamma g M$ is closed in $G$, we define $Q^{g} \subseteq H^{-\infty}\left(\sigma^{g}\right)$ to be the set of $\Gamma \cap g \mathrm{Mg}^{-1}$ invariant elements. Thus $Q^{g}$ is nonzero iff $\sigma^{g}$ occurs in

$$
\Gamma^{2}\left(g M g^{-1} / \Gamma \cap g M g^{-1}\right)
$$

and $\operatorname{dim}\left(Q^{g}\right)=$ multiplicity of $\sigma^{g}$ in

$$
L^{2}\left(g M g^{-1} / \Gamma \cap g M g^{-1}\right) .
$$

For each $g$ such that $Q^{g}$ is nonzero we can define a lift map $T_{g}: Q^{g} \rightarrow H^{-\infty}(\pi)$. Recalling that we are identifying $H^{\infty}(\pi)$ with $\mathscr{S}(G / M) \hat{\otimes} H^{\infty}(\sigma)$, given $\phi \in$ $\mathscr{S}(G / M) \hat{\otimes} H^{\infty}(\sigma), D \in Q^{g}$, define $T_{g}(D) \in H^{-\infty}(\pi)$ by

$$
T_{g}(D)(\phi)=\sum_{\gamma \in \Gamma / \Gamma \cap g M g^{-1}} D(\phi(\gamma \cdot g)) .
$$


The corollary to the Howe-Richardson theorem can be stated as

COROLlaRY 1.1. Let $S$ be a set of representatives for those $\Gamma: M$ double cosets such that (i) $\Gamma_{g} M$ is closed in $G$ and (ii) $Q^{g}$ is nonzero. Define $T\left(\oplus_{g \in S} Q^{g}\right) \rightarrow H^{-\infty}(\pi)$ by

$$
T\left(\bigoplus_{g \in S} D_{g}\right)=\sum_{g \in S} T_{g}\left(D_{g}\right) .
$$

Then $T$ is a linear isomorphism of $\bigoplus_{g \in S} Q^{g}$ onto the subspace of $\Gamma$ invariant elements in $H^{-\infty}(\pi)$.

2. Let $\pi$ be an irreducible representation of a connected simply connected nilpotent Lie group $N$. We assume that $N$ is a closed normal subgroup of a Lie group $G$ such that $G$ fixes the class of $\pi$. Let $\Gamma \subseteq G$ be a discrete cocompact subgroup of $G$ and $\Delta=\Gamma \cap N$. We assume $\Delta$ is cocompact in $N$, a condition that is automatic if $N$ is the nilradical of $G$ by a theorem of Mostow [Mo]. Suppose $\sigma$ is an irreducible representation of $G$ that restricts to a multiple of $\pi$ on $N$. We want to compute the multiplicity of $\sigma$ in ind ${ }_{\Gamma}^{G}(1)$ in terms of the multiplicity of $\pi$ in ind ${ }_{\Delta}^{N}(1)$ and the corresponding problem for the little group $G / N$.

Since $G$ stabilizes the representation $\pi$ of $N$, there exist a 2-cocycle $\alpha$ with values in $T=\left\{z \in \mathbf{C}^{x}|| z \mid=1\right\}$ such that $\pi$ extends to an $\alpha$-projective representation of $G$. If we denote by $\bar{\pi}$ this extension we have for $g_{1}, g_{2} \in G, \bar{\pi}\left(g_{1}\right) \bar{\pi}\left(g_{2}\right)=$ $\alpha\left(g_{1}, g_{2}\right) \bar{\pi}\left(g_{1} \cdot g_{2}\right)$. This 2-cocycle gives rise to a group extension and the Mackey obstuction can be viewed in this context $[\mathbf{A}-\mathbf{M}]$. Let $\bar{G}=T \times G$ as a Borel space and put a multiplication on $\bar{G}$ by $\left(t_{1}, g_{1}\right)\left(t_{2}, g_{2}\right)=\left(t_{1} t_{2} \alpha\left(g_{1}, g_{2}\right), g_{1} g_{2}\right)$. Then $\bar{G}$ is a Borel group and there exists a unique locally compact topology on $\bar{G}$ that turns $\bar{G}$ into a Lie group. Since the cocycle $\alpha$ may be taken to be identically one on $N$, we can view $N$ as embedded in $\bar{G}$ as the subgroup $\{(1, N) \mid n \in N\} \subseteq \bar{G}$ [K-L-I]. Now we see that $\bar{\pi}$ gives an ordinary representation of $\bar{G}$ by $\bar{\pi}(t, g)=t^{-1} \bar{\pi}(g)$.

If $\sigma$ is an $\alpha^{-1}$ projective representation of $G / N$, then $\sigma$ lifts to an $\alpha^{-1}$ projective representation of $G$ which is trivial on $N$. We can then view $\sigma$ as a representation of $\bar{G}$ by $\sigma(t, g)=t^{-1} \cdot \sigma(g)$. If we form $\bar{\pi} \otimes \sigma$, then $\bar{\pi} \otimes \sigma$ is a representation of $\bar{G}$ which is trivial on $T$, whence defines a representation of $\bar{G} / T=G$.

THEOREM 2.1. With notation as above, if $H^{\infty}(\pi), H^{\infty}(\sigma)$ are the $C^{\infty}$-vectors for $\pi$ and $\sigma$ respectively, then $H^{\infty}(\bar{\pi} \otimes \sigma)=H^{\infty}(\bar{\pi}) \hat{\otimes} H^{\infty}(\sigma)$, where $\hat{\otimes}$ is the projective tensor product.

For the proof see [Ro].

Let $\bar{\Gamma}$ be the inverse image of $\Gamma$ in $\bar{G}$ and, as above, view $\Delta$ as a subgroup of $\bar{\Gamma}$. Clearly $\bar{\Gamma}$ is a cocompact subgroup of $\bar{G}$ and $\Delta$ is a normal subgroup of $\bar{\Gamma}$.

Definition 2.1. Let $\Delta(\pi)=\left\{D \in H^{-\infty}(\pi) \mid \pi(\delta) D=D\right.$ for every $\left.\delta \in \Delta\right\}$.

LEMMA 2.1. $\bar{\pi}(\bar{\Gamma})$ acts in $\Delta(\pi)$ as a finite dimensional unitary representation of $\bar{\Gamma}$ with respect to the natural inner product on $\Delta(\pi)$.

Proof. By Theorem 2.1, if $\sigma$ is the trivial representation of $G / N$, then $H^{\infty}(\bar{\pi})=$ $H^{\infty}(\pi)$. Since $\Delta$ is a normal subgroup of $\bar{\Gamma}$ we do have an action of $\bar{\Gamma}$ on $\Delta(\pi)$. To see that the action is unitary it is enough to check it on elements of the form $(1, \gamma) \in \bar{\Gamma}$. 
Let $D, E \in \Delta(\pi), u, v \in H^{\infty}(\pi)$ and $\langle$,$\rangle the inner product on H(\pi)$. The definition of the inner product on $\Delta(\pi)$ gives

$$
\begin{aligned}
(\bar{\pi}(\gamma) D, E) \cdot\langle u, v\rangle & =\int_{N / \Delta} D\left(\pi\left(\bar{\gamma}^{-1}\right) \cdot \pi(n) u\right) E \overline{(\bar{\pi}(n) v)} d n \\
= & \int_{\Gamma N / \Gamma} D\left(\bar{\pi}\left(\gamma^{-1} \cdot n\right) \cdot u\right) \overline{E(\pi(n) v)} d n \\
= & \int_{\Gamma N / \Gamma} D(\pi(n) u) \overline{E(\bar{\pi}(\gamma n) v)} d n=\left(D, \bar{\pi}\left(\gamma^{-1}\right) E\right) \cdot\langle u, v\rangle .
\end{aligned}
$$

So $\bar{\pi}(\gamma)^{*}=\bar{\pi}\left(\gamma^{-1}\right)$ and thus $\bar{\pi}$ is a unitary representation of $\bar{\Gamma}$ on $\Delta(\pi)$. Q.E.D.

Lemma 2.2. Suppose $D \in H^{-\infty}(\bar{\pi} \otimes \sigma)$ is invariant under $\Gamma$. Then $D \in \Delta(\pi) \otimes_{\mathbb{C}}$ $H^{-\infty}(\sigma)$.

Proof. First note that we can consider the algebraic tensor product $\Delta(\pi) \otimes_{\mathbf{C}}$ $H^{-\infty}(\sigma)$ as a subspace of $H^{-\infty}(\bar{\pi} \otimes \sigma)$. Given $E \in \Delta(\pi), F \in H^{-\infty}(\sigma)$, define $(E \otimes F)(u, v)=E(u) F(v)$. Then $E \otimes F$ is a jointly continuous bilinear form on $H^{\infty}(\pi) \times H^{\infty}(\sigma)$, so by definition of the projective tensor product it extends to a continuous linear functional on $H^{\infty}(\pi) \hat{\otimes} H^{\infty}(\sigma)$.

Suppose $D \in H^{-\infty}(\bar{\pi} \otimes \sigma)$ is $\Gamma$ invariant. Let $E_{1}, \ldots, E_{L}$ be a basis of $\Delta(\pi)$. If we fix $u \in H^{\infty}(\sigma)$, then $D_{u}(x)=D(x \otimes u)$ is a $\Delta$ invariant element in $H^{-\infty}(\pi)$, so $D_{u}=\sum_{i=1}^{L} \lambda_{i}(u) E_{i}$. If we fix an $x \in H^{\infty}(\pi)$ we have $u \rightarrow D_{u}(x)=D(x \otimes u)$ is continuous, whence we get $\lambda_{i} \in H^{-\infty}(\sigma)$ for $i=1, \ldots, L$. It follows that on elementary tensors

$$
D(x \otimes u)=\sum_{i=1}^{L}\left(\lambda_{i} \otimes E_{i}\right)(x \otimes u),
$$

so by continuity we have

$$
D=\sum_{i=1}^{L} \lambda_{i} \otimes E_{i} \in \Delta(\pi) \otimes H^{-\infty}(\sigma) . \quad \text { Q.E.D. }
$$

Since $\bar{\pi}$ gives a unitary representation of $\bar{\Gamma}$ on $\Delta(\pi)$, we can decompose $\Delta(\pi)$ into a direct sum of irreducibles,

$$
\Delta(\pi)=\sum_{\alpha \in I} V(\alpha)
$$

Here $\alpha$ is an irreducible representation of $\bar{\Gamma} / \Delta$ on $V(\alpha)$, and $I$ is the indexing set.

$$
\Delta(\pi) \otimes_{\mathbb{C}} H^{-\infty}(\sigma)=\sum_{\alpha \in I}\left(V(\alpha) \otimes_{\mathbb{C}} H^{-\infty}(\sigma)\right),
$$

and each summand is invariant under $(\pi \otimes \sigma)(\bar{\Gamma})$. Hence we get

$$
\begin{aligned}
\operatorname{Hom}_{\Gamma}\left(1, \Delta(\pi) \otimes_{\mathbb{C}} H^{-\infty}(\sigma)\right) & =\operatorname{Hom}_{\bar{\Gamma}}\left(1, \Delta(\pi) \otimes_{\mathbb{C}} H^{-\infty}(\sigma)\right) \\
& =\bigoplus_{\alpha \in I} \operatorname{Hom}_{\bar{\Gamma}}\left(1, V(\alpha) \otimes_{\mathbb{C}} H^{-\infty}(\sigma)\right) .
\end{aligned}
$$


Recall that $\alpha$ is an irreducible representation of $\bar{\Gamma}$ which is trivial on $\Delta=\bar{\Gamma} \cap N$ and $\sigma$ is an irreducible representation of $\bar{G}$ which is trivial on $N$. Thus we can view $\alpha$ as a representation of $\bar{\Gamma} N / N$ and $\sigma$ as a representation of $\bar{G} / N$. Set $\Sigma=\bar{\Gamma} N / N$ and $E=\bar{G} / N$. Then we have the following isomorphisms

$$
\begin{aligned}
\operatorname{Hom}_{\bar{\Gamma}}\left(1, V(\alpha) \otimes H^{-\infty}(\sigma)\right) & \simeq \operatorname{Hom}_{\Sigma}\left(1, V(\alpha) \otimes H^{-\infty}(\sigma)\right) \\
& \simeq \operatorname{Hom}_{\Sigma}\left(V(\alpha)^{*}, H^{-\infty}(\sigma)\right) .
\end{aligned}
$$

If we apply Frobenius reciprocity to this last term we have

$$
\operatorname{Hom}_{\Sigma}\left(V(\alpha)^{*}, H^{-\infty}(\alpha)\right) \simeq \operatorname{Hom}_{E}\left(\sigma, \operatorname{ind}_{\Sigma}^{E}\left(\alpha^{*}\right)\right) .
$$

Therefore we have

THEOREM 2.2. With notation as above

$$
\begin{aligned}
\operatorname{dim}\left(\operatorname{Hom}_{\Gamma}\left(1, H^{\infty}(\bar{\pi} \otimes \sigma)\right)\right) \\
=\sum_{\alpha \in(\bar{\Gamma} N / N)^{-}} \operatorname{dim}\left(\operatorname{Hom}_{\bar{\Gamma} N}\left(\bar{\pi}, \operatorname{ind}_{\bar{\Gamma}} \overline{\bar{\Gamma}} N(\sigma)\right)\right) \cdot \operatorname{dim}\left(\operatorname{Hom}_{E}\left(\sigma, \operatorname{ind}_{\Sigma}^{E}(\alpha)\right)\right) .
\end{aligned}
$$

As it stands, this formula is difficult to use because of the group extensions $\bar{G}, \bar{\Gamma}$, etc. However, in [H-2] Howe has given a very succinct description of the Mackey obstruction when $N$ is nilpotent. Staying with the above situation ( $N \subseteq G$ as a normal subgroup, $\pi \in \hat{N}$ left fixed by $G$, and $\pi$ occurring with positive multiplicity in $\operatorname{ind}_{\Delta}^{N}(1)$ ), Howe [H-2, Proposition 2.3] has shown we may find a subgroup $R \subseteq G$ and a representation $\tau$ of $R \cap N=M$ such that

(i) $R / R \cap \Gamma$ and $M / M \cap \Delta$ are compact;

(ii) $G=R \cdot N$;

(iii) $\pi=\operatorname{ind}_{M}^{N}(\tau)$;

(iv) $\tau$ is $R$ invariant (of course $M$ is normal in $R$ );

(v) $M$ is connected;

(vi) if $M^{\prime}$ is the connected component of the identity of the kernel of $\tau$ then $M / M^{\prime}$ is a Heisenberg group or isomorphic to $\mathbb{R}$.

In what follows we will assume we are in a situation where (i)-(v) hold (but not necessarily (vi)). Suppose $\omega$ is an irreducible representation of $R$ that restricts to a multiple of $\tau$. Thus $\omega=\bar{\tau} \otimes \sigma$, where $\bar{\tau}$ is an $\alpha$-representation of $R$ and $\sigma$ is an $\alpha^{-1}$ representation of $R / M$. (As before, we are assuming $\alpha$ has been normalized to be trivial on $M$.) Since $R \cdot N=G$ and $\alpha$ is trivial on $R \cap N$, we can extend $\alpha$ to a cocycle of $G / N$. We can similarly view $\sigma$ as an irreducible $\alpha$ representation of $G / N$. Now $\pi=\operatorname{ind}_{M}^{N}(\tau)$ acts on the Hilbert space

$$
H(\pi)=\left\{\phi: N \rightarrow H(\tau) \mid \phi(n m)=\tau\left(m^{-1}\right) \phi(n) \text { and } \int_{N / M}|\phi(n)|^{2} d n<\infty\right\} .
$$

Since $G=R \cdot N$, we can define a projective extension of $\pi, \bar{\pi}$, by defining $\bar{\pi}$ on $R$. In this case we have

$$
(\bar{\pi}(r) \phi)(n)=\bar{\tau}(r) \phi\left(r^{-1} n r\right) .
$$


It is now easy to see that $\bar{\pi} \otimes \sigma$ is an ordinary representation of $G$ acting on $H(\pi) \otimes H(\sigma)$ and that $\bar{\pi} \otimes \sigma$ is equivalent to ind ${ }_{R}^{G}(\bar{\tau} \otimes \sigma)$.

Now recall from $\S 1$ that if $S$ is the set of $\Delta n M$ double cosets such that (1) $\Delta n M$ is closed, and (2) $\tau^{n}$ occurs with positive multiplicity in $\operatorname{ind}_{\Delta \cap n M n_{-1}}^{n M n^{-1}}$ (1), then we described

$$
\Delta(\pi)=\left\{D \in H^{-\infty}(\pi) \mid \pi(\delta) D=D \text { for every } \delta \in \Delta\right\}
$$

in terms of the Richardson lift maps. If

$$
Q^{n}=\left\{D \in H^{-\infty}\left(\tau^{n}\right) \mid \tau^{n}(\gamma) D=D \text { for every } \gamma \in \Delta \cap n M n^{-1}\right\}
$$

then we had $T^{n}: \mathbf{Q}^{n} \rightarrow \Delta(\pi)$. If we formed the direct sum we had $\oplus_{n \in S} T^{n}$ : $\bigoplus_{n \in S} \mathbf{Q}^{n} \rightarrow \Delta(\pi)$ is an isomorphism.

For each $n \in S$ we can extend $T^{n}$ by $T^{n} \otimes 1$ as a map from $\mathbf{Q}^{n} \otimes H^{-\infty}(\sigma)$ to $\Delta(\pi) \otimes H^{-\infty}(\sigma)$. If no confusion is likely to arise we will denote $T^{n} \otimes 1$ by $T^{n}$ also.

Let $D \otimes E \in Q^{n} \otimes H^{-\infty}(\sigma)$. We want to compute $(\bar{\pi} \otimes \sigma)(\gamma)\left(T^{n}(D \otimes E)\right)$ for $\gamma \in \Gamma$. Since $G=R \cdot N$ we can find $s \in N, r \in R$ so that $\gamma \cdot n=s \cdot r$. Now let $\phi \otimes u \in H^{\infty}(\pi) \otimes H^{\infty}(\sigma)$. We have

$$
\begin{aligned}
{\left[(\bar{\pi} \otimes \sigma)(\gamma)\left(T^{n}(D \otimes E)\right)\right](\phi} & \otimes u)=T^{n}(D \otimes E)\left(\pi\left(\gamma^{-1}\right) \phi \otimes \sigma\left(\gamma^{-1}\right) u\right) \\
& =\sum_{\delta \in \Delta / \Delta \cap n M n^{-1}}(D \otimes E)\left(\bar{\pi}\left(\gamma^{-1}\right) \phi(\delta n) \otimes \sigma\left(\gamma^{-1}\right) u\right) \\
& =\sum_{\delta \in \Delta / \Delta \cap n M n^{-1}} D(\phi(\gamma \cdot \delta \cdot n)) \cdot E\left(\sigma\left(\gamma^{-1}\right) \cdot u\right) \\
& =\sum_{\delta \in \Delta / \Delta \cap n M n^{-1}} D\left(\phi\left(\gamma \delta \gamma^{-1} \cdot \gamma n\right)\right) \cdot(\sigma(\gamma) E)(u) .
\end{aligned}
$$

Recall that $\gamma n=s \cdot r$ with $r \in R$ and $s \in N$, so using the transformation properties of $\phi$ we have that (2.8) equals

$$
\sum_{\Delta \cap \gamma n M n^{-1} \gamma^{-1}} D\left(\bar{\tau}\left(r^{-1}\right) \phi(\delta s)\right)(\sigma(\gamma) E)(u) .
$$

Set $\bar{D}(u)=D\left(\bar{\tau}\left(r^{-1}\right) \cdot u\right)$ for $u \in H^{\infty}(\tau)$. Noting that $\gamma n M n^{-1} \gamma^{-1}=s r M r^{-1} s^{-1}$ $=s M s^{-1}$ (since $R$ normalizes $M$ ), we have that (2.9) can be written as

$$
\sum_{\Delta / \Delta \cap s M s^{-1}} \bar{D}(\phi(\delta s))(\sigma(\gamma) E(u))=\sum_{\delta \in \Delta / \Delta \cap s M s^{-1}}(\bar{D} \otimes \sigma(\gamma) E)(\phi(\delta s) \otimes u) .
$$

Now (2.10) will be of the form $T^{s}(\bar{D} \otimes \sigma(\gamma) E)$ for some $s \in S$ iff the following two conditions on $s$ hold.

(i) $\Delta s M$ is closed in $N$ and

(ii) $Q^{s}$ is nonzero.

Now $\Delta n M$ is closed iff $\gamma \Delta n M n^{-1} \gamma^{-1}=\gamma \Delta \gamma^{-1} \cdot \gamma n M n^{-1} \gamma^{-1}=\Delta s M s^{-1}$ is closed, whence (i) follows. As for (ii), $\bar{D}$ is certainly nonzero, so we compute for $\delta \in \Delta \cap$ $s M s^{-1}, u \in H^{\infty}(\tau)$

$$
\begin{aligned}
\left(\tau^{s}(\delta)(\bar{D})\right)(u) & =\bar{D}\left(\tau^{s}\left(\delta^{-1}\right) \cdot u\right)=D\left(\bar{\tau}\left(r^{-1}\right) \tau\left(s^{-1} \delta s\right) \cdot u\right) \\
& =D\left(\bar{\tau}\left(r^{-1} s^{-1} \delta s r\right) \bar{\tau}\left(r^{-1}\right) u\right)=D\left(\tau\left(n^{-1} \gamma^{-1} \delta \gamma n\right) \bar{\tau}\left(r^{-1}\right) u\right) \\
& =D\left(\tau^{n}\left(\gamma^{-1} \delta \gamma\right) \cdot \bar{\tau}\left(r^{-1}\right) u\right)=D\left(\bar{\tau}\left(r^{-1}\right) u\right)=\bar{D}(u)
\end{aligned}
$$


In the last line we have used that $\gamma^{-1} \delta \gamma \in \Delta \cap n M n^{-1}$ and that $D \in Q^{n}$. Therefore $s$ defines a class in $S$, which we will write as $[s]$.

Lemma 2.3. For each $\gamma \in \Gamma$, define a map $\gamma: S \rightarrow S$ by $\gamma \cdot[n]=[s]$. This defines a group action on $S$.

Proof. We first check that the map $\gamma: S \rightarrow S$ is well defined. If $n \in[n]$, and we have $\gamma \cdot n=s \cdot r=s^{\prime} \cdot r^{\prime}$, then $s^{\prime-1} \cdot s=r^{\prime} \cdot r^{-1} \in R \cap N=M$, so $\Delta s^{\prime} M=\Delta s M$. Now choose a different representative in $[n]$, say $\bar{n}=\delta \mathrm{nm}$. Then

$$
\gamma \cdot \bar{n}=\gamma \cdot \delta n m=\gamma \cdot n \cdot\left(n^{-1} \delta n \cdot m\right)=s \cdot r \cdot n^{-1} \delta n m
$$

where $s \in R, r n^{-1} \delta n m \in N$. By the above this defines the class $\Delta s M$. Thus $\gamma \cdot[n]=$ $[s]$ is well defined.

Let $\gamma_{1}, \gamma_{2} \in \Gamma$. Then $\gamma_{1} \cdot \gamma_{2}[n]=[s]$ where $\gamma_{1} \gamma_{2} n=s \cdot r$ if $\gamma_{2} n=s_{2} r_{2}$ and $\gamma_{1}\left(s_{2}\right)=s_{1} \cdot r_{1} \gamma_{1}\left[\gamma_{2}[n]\right]=\left[s_{1}\right]$. But $\gamma_{1} \gamma_{2} n=\gamma_{1}\left(s_{2} r_{2}\right)=s_{1} \cdot r_{1} r_{2}$ says that $\left[s_{1}\right]=$ $\gamma_{1} \gamma_{2}[n]$. So we have a group action. Q.E.D.

LEMMA 2.4. Given $[n] \in S$, the stabilizer of $[n]$ in $\Gamma$ is exactly $\left(n R n^{-1} \cap \Gamma\right) \cdot \Delta$.

Proof. If $\gamma \cdot[n]=[n]$ we have $\gamma n=s \cdot r$ and $s=\delta n m$ for $\delta \in \Delta, m \in M$. Thus $\gamma \cdot n=\delta n m r$ or $\gamma=\delta n m n^{-1} \in\left(n R n^{-1} \cap \Gamma\right) \cdot \Delta$. Clearly $\left(n R n^{-1} \cap \Gamma\right) \cdot \Delta$ stabilizes [n]. Q.E.D.

COROllaRY 2.1. With the notation as above, $\left(n \mathrm{Rn}^{-1} \cap \Gamma\right) \cdot \Delta$ has finite index in $\Gamma$, hence $R n \Gamma$ is closed in $G$ for every $n \in S$.

Proof. $S$ is a finite set, so every $\Gamma$ orbit is finite. Q.E.D.

LEMMA 2.5. The map $T^{n} \otimes 1: Q^{n} \otimes H^{-\infty}(\sigma) \rightarrow \Delta(\pi) \otimes H^{-\infty}(\sigma)$ intertwines the action of $\left(n R n^{-1} \cap \Gamma\right)$ on $Q^{n} \otimes H^{-\infty}(\sigma)$ and its image in $\Delta(\pi) \otimes H^{-\infty}(\sigma)$.

Proof. Recall that $\bar{\pi} \otimes \sigma=\operatorname{ind}_{R}^{G}(\bar{\tau} \otimes \sigma)$, so we can view $\phi \in H^{\infty}(\bar{\pi} \otimes \sigma)$ as a function $\phi: G \rightarrow H^{\infty}(\bar{\tau} \otimes \sigma)$ such that $\phi(g r)=(\bar{\tau} \otimes \sigma)\left(r^{-1}\right) \cdot \phi(g)$. If $\gamma \in\left(n R n^{-1}\right.$ $\cap \Gamma)$ and $D \otimes E \in Q^{n} \otimes H^{-\infty}(\sigma)$ we have

$$
\begin{aligned}
\left(T^{n} \otimes 1\right)\left((\bar{\tau} \otimes \sigma)^{n}(\gamma)\right. & (D \otimes E))(\phi) \\
= & \sum_{\delta \in \Delta / \Delta \cap n M n^{-1}}(D \otimes E)\left((\bar{\tau} \otimes \sigma)^{n}\left(\gamma^{-1}\right) \phi(\delta n)\right) .
\end{aligned}
$$

Now $(\bar{\tau} \otimes \sigma)^{n}\left(\gamma^{-1}\right)=(\bar{\tau} \otimes \sigma)\left(n^{-1} \gamma^{-} n\right)$. But $\gamma \in n R n^{-1} \cap \Gamma$ implies $n^{-1} \gamma^{-1} n \in$ $R$. Thus

$$
\begin{aligned}
(\bar{\tau} \otimes \sigma)^{n}\left(\gamma^{-1}\right) \cdot & \phi(\delta n)=(\tau \otimes \sigma)\left(n^{-1} \gamma^{-1} n\right) \cdot \phi(\delta n) \\
& =\phi\left(\delta n^{-1} \gamma n\right)=\phi\left(\gamma \cdot \gamma^{-1} \delta \gamma \cdot n\right)=\left((\bar{\pi} \otimes \sigma)\left(\gamma^{-1}\right) \phi\right)\left(\gamma^{-1} \delta \gamma n\right) .
\end{aligned}
$$

Thus (2.12) turns into

$$
\sum_{\delta \in \Delta / \Delta \cap \gamma^{-1} n M n^{-1} \gamma}(D \otimes E)(\bar{\pi} \otimes \sigma)\left(\gamma^{-1}\right)(\phi)(\delta n) .
$$


But $\gamma^{-1} \in n R n^{-1} \cap \Gamma$ implies that $\gamma^{-1} n M n^{-1} \gamma=n M n^{-1}$ so (2.13) becomes

$$
\begin{aligned}
& \sum_{\delta \in \Delta / \Delta \cap n M n^{-1}}(D \otimes E)\left((\bar{\pi} \otimes \sigma)\left(\gamma^{-1}\right)(\phi)(\delta n)\right) \\
&= T^{n}(D \otimes E)\left((\bar{\pi} \otimes \sigma)\left(\gamma^{-1}\right)(\phi)\right) \\
&=(\bar{\pi} \otimes \sigma)(\gamma)\left(T^{n}(D \otimes E)\right)(\phi) .
\end{aligned}
$$

Thus we have that $T^{n} \otimes 1$ intertwines $\left.(\bar{\tau} \otimes \sigma)^{n}\right|_{n R n^{-1} \cap \Gamma}$ acting on $Q^{n} \otimes H^{-\infty}(\sigma)$ and $\left.(\bar{\pi} \otimes \sigma)\right|_{n R n^{-1} \cap \Gamma}$ acting on $\Delta(\pi) \otimes H^{-\infty}(\sigma)$. Q.E.D.

We have that $\Delta(\pi) \otimes H^{-\infty}(\sigma)=\oplus_{n \in S} T_{n}\left(Q^{n} \times H^{-\infty}(\sigma)\right)$ and we want to decompose $\Delta(\pi) \otimes H^{-\infty}(\sigma)$ into $\Gamma$ invariant subspaces in a natural way. To this end, let $\Omega \in S / \Gamma$ be a $\Gamma$-orbit in $S$. If we fix $n \in \Omega$ and set $\Gamma_{n}=\left(n R n^{-1} \cap \Gamma\right) \cdot \Delta$ then $\Omega$ is isomorphic to $\Gamma / \Gamma_{n}$. Now we have a representation of $n R n^{-1} \cap \Gamma$ on $Q^{n} \otimes$ $H^{-\infty}(\sigma)$ and this representation is trivial on $\left(n R n^{-1} \cap \Gamma\right) \cap \Delta$. Thus we can extend it to a representation of $\left(n R n^{-1} \cap \Gamma\right) \cdot \Delta$ on $Q^{n} \otimes H^{-\infty}(\sigma)$. Let $\beta_{n}$ be this extended representation of $\Gamma_{n}$.

Let $V(\Omega)=\sum_{m \in \Omega}\left(T^{m}\left(Q^{m} \otimes H^{-\infty}(\sigma)\right)\right)$. Then $V(\Omega)$ is a $\Gamma$ invariant subspace of $\Delta(\pi) \otimes H^{-\infty}(\sigma)$ under the action $\bar{\pi} \otimes \sigma$. Thus $(\bar{\pi} \otimes \sigma, V(\Omega))$ is a representation of $\Gamma$ on $V(\Omega)$. If $n \in \Omega, \Gamma_{n}$ and $\beta_{n}$ are defined as above, then it is clear that $(\bar{\pi} \otimes \sigma, V(\Omega)) \simeq \operatorname{ind}_{\Gamma_{n}}^{\Gamma}\left(\beta_{n}\right)$. Consequently we have

$$
\Delta(\pi) \otimes H^{-\infty}(\sigma)=\bigoplus_{[n] \in S / \Gamma} \operatorname{ind}_{\Gamma_{n}}^{\Gamma}\left(\beta_{n}\right) .
$$

Therefore we have

$$
\operatorname{Hom}_{\Gamma}\left(1, \Delta(\pi) \otimes H^{-\infty}(\sigma)\right)=\bigoplus_{n \in S / \Gamma} \operatorname{Hom}_{\Gamma}\left(1, \operatorname{ind}_{\Gamma_{n}}^{\Gamma}\left(\beta_{n}\right)\right) .
$$

If we apply Frobenius reciprocity to (2.16) we get

$$
\operatorname{Hom}_{\Gamma}\left(1, \operatorname{ind}_{\Gamma_{n}}^{\Gamma}\left(\beta_{n}\right)\right) \simeq \operatorname{Hom}_{\Gamma_{n}}\left(1, \beta_{n}\right)
$$

which implies that

$$
\operatorname{Hom}_{\Gamma}\left(1, \Delta(\pi) \otimes H^{-\infty}(\sigma)\right)=\bigoplus_{n \in S / \Gamma} \operatorname{Hom}_{\Gamma_{n}}\left(1, \beta_{n}\right) .
$$

Now Theorem 0.1 says

$$
\operatorname{Hom}_{\Gamma}\left(1, \Delta(\pi) \otimes H^{-\infty}(\sigma)\right) \simeq \operatorname{Hom}_{G}\left(\bar{\pi} \otimes \sigma, \operatorname{ind}_{\Gamma}^{G}(1)\right)
$$

and

$$
\operatorname{Hom}_{\Gamma_{n}}\left(1, \beta_{n}\right) \simeq \operatorname{Hom}_{n R n^{-1}}\left(\tau^{n} \otimes \sigma, \operatorname{ind}_{n R n^{-1} \cap \Gamma}^{n R n^{-1}}(1)\right) .
$$

Finally if we identify $S / \Gamma$ as the closed $R n \Gamma$ double cosets such that

$$
\operatorname{Hom}_{n R n^{-1}}\left((\bar{\tau} \otimes \sigma)^{n}, \operatorname{ind}_{n R n^{-1} \cap \Gamma}^{n R n^{-1}}(1)\right) \neq 0,
$$

then we can summarize the above discussion with

THEOREM 2.3. With notation as above, if $\lambda=\bar{\pi} \otimes \sigma=\operatorname{ind}_{R}^{G}(\bar{\tau} \otimes \sigma)$ then

$$
\operatorname{Hom}_{G}\left(\lambda, \operatorname{ind}_{\Gamma}^{G}(1)\right) \simeq \bigoplus_{\substack{R n \Gamma \\ \text { closed double coset }}} \operatorname{Hom}_{n R n^{-1}}\left((\tau \otimes \sigma)^{n}, \operatorname{ind}_{n R n^{-1} \cap \Gamma}^{n R n^{-1}}(1)\right) \text {. }
$$


3. In this section we give an application of Theorem 2.3 to solvable Lie groups to obtain a multiplicity formula in terms of Penney's canonical objects. Let $G$ be a connected simply connected solvable Lie group with $\Gamma \subseteq G$ a discrete cocompact subgroup. Let $N \subseteq G$ be the nilradical of $G$ and $\Delta=N \cap \Gamma$. As we have seen, $\Delta$ is cocompact in $N$. We first state a very general result of Howe [H-1].

THEOREM 3.1. Let $G$ be a locally compact group and $\Gamma \subseteq G$ a discrete cocompact subgroup. Let $E \subseteq G$ be a normal subgroup such that $E \cap \Gamma$ is cocompact in $E$. Let $\sigma$ be an invariant representation of $E$ such that $\rho=\operatorname{ind}_{H}^{G}(\sigma)$ is an irreducible representation of $G$. Let $m(\rho)$ denote the multiplicity of $\rho$ in $L^{2}(G / \Gamma)$ and $m\left(\sigma^{g}\right)$ denote the multiplicity of $\sigma^{g}$ in $L^{2}(H / H \cap \Gamma)$. Then the number of double cosets $H g \Gamma$ such that $m\left(\sigma^{g}\right)>0$ is finite and we have

$$
m(\rho)=\sum_{g \in \Gamma / G \backslash H} m\left(\sigma^{g}\right) .
$$

Now we need to describe the canonical objects in Kirillow theory constructed by Penney [P-5]. Let $N$ be a connected simply connected nilpotent Lie group with Lie algebra $\underline{\mathbf{N}}$. Let $f \in \underline{\mathbf{N}}^{*}$ and $\pi$ the irreducible representation of $N$ associated to $f$ via Kirillov theory. Let $\underline{\mathbf{R}}(f)$ be the radical of the two-form $B_{f}(X, Y)=f([X, Y])$. Let $\underline{\mathbf{H}}_{1}(f)$ be the ideal generated by $\underline{\mathbf{R}}(f)$ and define inductively $\underline{\mathbf{H}}_{k+1}(f)=\underline{\mathbf{H}}_{1}\left(\left.f\right|_{\underline{\mathbf{H}}_{k}}\right)$. Set $\underline{\mathbf{H}}(f)=\bigcap_{k} \underline{\mathbf{H}}_{k}(f)$ and $\underline{\mathbf{K}}(f)=\underline{\mathbf{H}}^{B}(f)=\left\{x \in \underline{\mathbf{N}} \mid B_{f}(x, \underline{\mathbf{H}}(f))=0\right\}$. In [P-5] Penney shows the following.

LEMMA 3.1. (a) If $K(f)$ is the connected subgroup of $N$ corresponding to $\underline{\mathbf{K}}(f)$ and $\omega$ is the representation of $K(f)$ corresponding to $\left.f\right|_{\underline{\mathbf{K}}(f)}$, then $\operatorname{ind}_{K(f)}^{N}(\omega)$ is equivalent to $\pi$.

(b) Let $A$ be an automorphism of $N$ (and denote by $A$ the action on $\underline{\mathbf{N}}$ and $\underline{\mathbf{N}}^{*}$ also). Then $A \cdot(K(f))=K(A(f))$. In particular, $K(f)$ is stabilized by any automorphism of $N$ which fixes $f$.

(c) $\omega$ is square-integrable modulo its kernel.

We need to recall a small amount of how one constructs an irreducible representation from an integral element $f \in \underline{\mathbf{G}}^{*}$. Let $f \in \underline{\mathbf{G}}^{*}$ and $G(f)=\left\{g \in G \mid \operatorname{Ad}^{*}(g) f\right.$ $=f\}$. Recall that $f$ is called integral if there exists a character $\eta$ of $G(f)$ such that $d \eta=\left.2 \pi i f\right|_{\underline{\mathbf{G}}(f)}$. Given an integral element $f \in \underline{\mathbf{G}}^{*}$ and a character $\eta$ of $G(f)$ one can construct an irreducible representation $\rho(f, \eta)$ of $G[\mathbf{M}]$.

Let $l=\left.f\right|_{\underline{\mathbf{N}}}$ and let $\pi(l)$ be the irreducible representation of $N$ associated to $l$ via Kirillov theory. If $G(l)=\left\{g \in G \mid \operatorname{Ad}^{*}(g)(l)=l\right\}$ then there is a canonical extension of $\pi$ to $G(l) \times{ }_{s} N$ given by $\bar{\pi}(g, n)=\nu(g) \pi(n)$. With the data $(f, \eta)$, a representation $\sigma_{f}$ of $G(l)$ is constructed such that $\sigma_{f} \times \bar{\pi}$ drops to a representation of $G(l) N$. One then has that $\rho\left(f, \eta_{f}\right)=\operatorname{ind}_{G(l) N}^{G}\left(\sigma_{f} \otimes \bar{\pi}\right)$ is the desired irreducible representation of $G$. If $\rho$ is a type I representation of $G$, then there is an integral $f \in \underline{\mathbf{G}}^{*}$ and $\eta$ such that $\rho \simeq \rho(f, \eta)$ (see [M]). In particular every representation occurring in $L^{2}(G / \Gamma)$, being CCR, occurs this way. 
Now fix a representation $\rho(f, \eta)$ of $G$ and set $H=G(l) \cdot K(l)$ where $l=\left.f\right|_{\underline{N}}$. Then we have $\bar{\omega}=\nu \otimes \omega$ is an extension of $\omega$ to $G(l) \times{ }_{s} K(l)$ and $\sigma_{f} \otimes \omega$ is a representation of $H=G(l) \cdot K(l)$. From Lemma 3.1(a) it is clear that ind ${ }_{H}^{G}\left(\sigma_{f} \otimes \bar{\omega}\right)$ $=\rho(f, \eta)$.

THEOREM 3.2. Let $\tau=\sigma_{f} \otimes \bar{\omega}$ and $\rho(f, \eta)=\operatorname{ind}_{H}^{G}(\tau)$. If $m(\rho)$ is the multiplicity of $\rho$ in $L^{2}(G / \Gamma)$ then

$$
m(\rho)=\sum_{\substack{g \in \Gamma \backslash G / H \\ \text { closed double cosets }}} m\left(\tau^{g}\right)
$$

where $m\left(\tau^{g}\right)=$ multiplicity of $\tau^{g}$ in ind ${ }_{g H g^{-1}}^{g H g}(1)$.

Proof. Recall that $\rho=$ ind $_{G(l) N}^{G}\left(\sigma_{f} \otimes \bar{\pi}\right)$ and $G(l) N$ is a normal subgroup of $G$. Consequently we can apply Theorem 3.1 to conclude that

$$
m(\rho)=\sum_{g \in \Gamma: G(l) N} m\left(\left(\sigma_{f} \otimes \bar{\pi}\right)^{g}\right)
$$

where $m\left(\left(\sigma_{f} \otimes \bar{\pi}\right)^{g}\right)$ is the multiplicity of $\left(\sigma_{f} \otimes \pi\right)^{g}$ in ind $\stackrel{\Gamma}{\cap}(l) N_{G(l) N}(1)$. Now it follows from Lemma 2.2 that if $m\left(\left(\sigma_{f} \otimes \bar{\pi}\right)^{g}\right)>0$, then $\pi^{g}$ occurs with positive multiplicity in $L^{2}(N / \Delta)$. By relabeling we may as well assume that $\pi$ occurs in $L^{2}(N / \Delta)$. Now $\pi^{g}$ corresponds to $\mathrm{Ad}^{*}(g)(l)$ under Kirillov correspondence, so if we replace $g$ by $g \cdot n$ for some $n \in N$, we can assume that we have chosen coset representatives $g_{1}, \ldots, g_{L}$ so that $\operatorname{Ad}^{*}\left(g_{i}\right)(l)$ is a rational element of $\underline{\mathbf{N}}^{*}$.

Now we claim if $l \in \underline{\mathbf{N}}^{*}$ is a rational element, then $G(l) \cdot K(l) \cap \Gamma$ is cocompact in $G(l) \cdot K(l)$. First note that if $l \in \underline{\mathbf{N}} *$ is rational then $\underline{\mathbf{K}}(l)$ is rational, so $K(l) \cap \Gamma$ is cocompact in $K(l)$. Now let $L \subseteq \underline{\mathbf{N}}$ be the lattice generated by $\log (\Delta)$. Since $\Gamma$ normalized $\Delta, \operatorname{Ad}(\gamma)(L) \subseteq L$ for all $\gamma \in \Gamma$. If $L^{\perp}=\left\{f \in \underline{\mathbf{N}}^{*} \mid f(L) \subseteq \mathbb{Z}\right\}$, then by duality $\operatorname{Ad}^{*}(l)\left(L^{\perp}\right) \subseteq L^{\perp}$ for all $\gamma \in \Gamma$. If $l \in \underline{\mathbf{N}}^{*}$ is rational, then $c \cdot l \in L^{\perp}$ for some integer $c$, and $G(c \cdot l)=G(l)$. Now $\operatorname{Ad}^{*}(\Gamma)(c \cdot l) \subseteq L^{\perp}$, and $L^{\perp}$ is a discrete, hence closed, subset of $\underline{\mathbf{N}}^{*}$. It follows that $\Gamma \cdot G(c \cdot l)=\Gamma \cdot G(l)$ is closed in $G$, whence $\Gamma \cap G(l)$ is cocompact in $G(l)$. Now consider a sequence $x_{n} y_{n} \gamma_{n}$ converging to $g \in G$ with $x_{n} \in G(l), y_{n} \in K(l)$, and $\gamma_{n} \in \Gamma$. By passing to a subsequence and using compactness of $G(l) / G(l) \cap \Gamma$ we can find $\delta_{n} \in G(l) \cap \Gamma$ so that $x_{n} \delta_{n}$ converges in $G(l)$. Then $\left(x_{n} \delta_{n}\right)\left(\delta_{n}^{-1} y_{n} \delta_{n}\right) \cdot \delta_{n}^{-1} \gamma_{n}$ converges to $g \in G$, so $\delta_{n}^{-1} y_{n} \delta_{n} \gamma_{n}$ converges in $G$. Since $K(l) \cdot \Gamma$ is closed, it follows that $\left(x_{n} \delta_{n}\right)\left(\delta_{n}^{-1} y_{n} \delta_{n} \gamma_{n}\right)$ converges to an element in $G(l) K(l) \Gamma$. Therefore, $G(l) K(l) \cdot \Gamma$ is closed in $G$, so $G(l) K(l) \cap \Gamma$ is cocompact in $G(l) K(l)$.

Applying the above claim to $\operatorname{Ad}^{*}\left(g_{i}\right)(l)$ and using the fact that $g_{i}(G(l) K(l)) g_{i}^{-1}$ $=G\left(\operatorname{Ad}^{*}\left(g_{i}\right)(l)\right)$, we conclude that for $g_{1}, \ldots, g_{L} g_{i} H g_{i}^{-1} \cap \Gamma$ is cocompact in $g_{i} H g_{i}^{-1}$. Since ind $g_{i}^{G(l) g_{i}^{-1}}\left(\tau^{g}\right)=\left(\sigma_{f} \times \bar{\pi}\right)^{g}$, we can use Theorem 2.2 to conclude that

$$
m\left(\left(\sigma_{f} \otimes \bar{\pi}\right)^{g}\right)=\sum_{\substack{n \in\left(g_{i} H g_{i}^{-1}\right): \Gamma \cap(G(l) \cdot N) \\ \text { closed double cosets }}} m\left(\tau^{g}\right)
$$

where $m\left(\tau^{g}\right)=$ multiplicity of $\tau^{g}$ in ind ${ }_{g g^{-1}}^{g g^{-1}} \cap \Gamma(1)$.

Combining (3.4) with (3.3) gives the desired results. Q.E.D. 
ACKNOWLEDGMENT. The author would like to thank the referee for his many detailed remarks on this paper. Both the paper and the author benefited greatly from his comments.

\section{REFERENCES}

[A-M] L. Auslander and C. Moore, Unitary representations of solvable Lie groups, Mem. Amer. Math. Soc. No. 62 (1966).

[C-G-1] L. Corwin and F. Greenleaf, Character formula and spectra of compact nilmanifolds, J. Funct. Anal. 21 (1976), 123-154.

[C-G-2] _ Intertwining operators for representations induced from uniform subgroups, Acta Math. 136 (1976), 275-301.

[C-G-P] L. Corwin, F. Greenleaf, and R. Penney, A general character formula for irreducible projections on $L^{2}$ of a nilmanifold, Math. Ann. 225 (1977), 21-32.

[G] I. Gelfand, M. Graev, and I. Pyatetskii-Shapiro, Representation theory and automorphic functions, Saunders, Philadelphia, Pa., 1969.

[H-1] R. Howe, On Frobenius reciprocity for unipotent algebraic groups over $\mathbb{Q}$, Amer. J. Math. 93 (1971).

[H-2] $383-437$.

[H-3] _ On a condition between nilpotent groups and oscillatory integrals associated to singularities, Pacific J. Math. 73 (1977), 329-365.

[K-L-1] A. Kleppner and R. Lipsman, The Plancherel formula for group extensions. I, Ann. Sci. École Norm. Sup. (4) 5 (1972), 459-516.

[M] C. Moore, Representations of solvable and nilpotent groups and harmonic analysis on nil and solvmanifolds, Proc. Sympos. Pure Math., vol. 26, Amer. Math. Soc., Providence, R. I., 1973, pp. 3-45.

[Mo] G. Mostow, Factor spaces of solvable Lie groups, Ann. of Math. (2) 60 (1954), 1-27.

[P-1] R. Penney, Abstract Plancherel theorems and a Frobenius reciprocity theorem, J. Funct. Anal. 18 1975, 177-190.

[P-2] _ Spherical distributions on Lie groups and $C^{\infty}$-vectors, Trans. Amer. Math. Soc. 223 (1976), $368-384$.

[P-3] _ Decomposition of $C^{\infty}$-intertwining operators for Lie groups, Proc. Amer. Math. Soc. 54 (1976), 368-370.

[P-4] L Lie algebra cohomology of representations of nilpotent Lie groups and holomorphically induced representations, Trans. Amer. Math. Soc. 261 (1980), 33-51.

[P-5] _ Canonical objects in Kirillov theory on nilpotent Lie groups, Proc. Amer. Math. Soc. 66 (1977), 175-178.

[Po] N. Poulsen, On $C^{\infty}$-vectors and intertwining bilinear forms for representations of Lie groups, J. Funct. Anal. 9 (1972), 87-120.

[R] L. Richardson, Decomposition of the $L^{2}$-space of a general compact nilmanifold, Amer. J. Math. 93 (1971).

[Ro] J. Rosenberg, Realization of square integrable representations of unimodular Lie groups on $L^{2}$ cohomology spaces, Trans. Amer. Math. Soc. 26 (1980), 1-32.

Department of Mathematics, Louisiana State University, Baton Rouge, Louisiana 70803 